\title{
MACROLACTAMAS BIOATIVAS E SÍNTESE DE MACROCICLOS POR REAÇÃO DE CARBOCICLIZAÇÃO RADICALAR MEDIADA POR HIDRETO DE TRI- $N$-BUTILESTANHO
}

\author{
Renata Fontes Prado Faraco, Magno Carvalho Pires, Ana Paula Campos Rocha e Maria Auxiliadôra Fontes Prado* \\ Departamento de Produtos Farmacêuticos, Faculdade de Farmácia, Universidade Federal de Minas Gerais, Av. Presidente Antônio \\ Carlos 6.627, 31270-901 Belo Horizonte - MG, Brasil
}

Recebido em 27/2/07; aceito em 27/9/07; publicado na web em 13/8/08

BIOACTIVE MACROLACTAMS AND SYNTHESIS OF MACROCYCLES BY TRI- $N$-BUTYLTIN HYDRIDE-MEDIATED RADICAL CARBOCYCLIZATION REACTION. This review presents natural, semi-synthetic and synthetic bioactive macrolactams and their structure-activity relationships when available. For macrolactams in clinical use the advantages and disadvantages in relation to other drugs are presented, and for synthetic macrolactams the method used in the cyclization is showed. Regarding macrocyclic synthesis by the tri- $n$-butyltin hydride-mediated radicalar carbocyclization reaction the precursor, the reaction conditions, products and yields, mechanism and cyclization mode are discussed.

Keywords: bioactive macrolactams; radical carbocyclization; macrocyclization.

\section{INTRODUÇÃO}

Os macrociclos apresentam importantes atividades biológicas e muitos deles, ou seus derivados, são usados como fármacos (eritromicina, ciclosporina, vancomicina e anfotericina B) ${ }^{1-3}$ As atividades biológicas dos macrociclos, substâncias com enorme variedade estrutural, são atribuídas, em parte, a uma característica comum: a restrição conformacional em relação a compostos de cadeia aberta. ${ }^{2,4}$

Entre os macrociclos que são agentes bioativos encontram-se as macrolactamas, cujas atividades biológicas se devem, em parte, ao fato de serem macrociclos e também amidas. Em um trabalho publicado recentemente, no qual são apresentados dados relativos a 128 candidatos a fármacos em desenvolvimento em três grandes indústrias farmacêuticas, é relatado que $12 \%$ das reações realizadas na síntese das 128 substâncias são de acilação, especialmente $N$-acilações. Os autores não se surpreendem com esse dado, uma vez que as amidas estão presentes em um grande número de fármacos. Além disso, de 53 fármacos que, em 2003, foram responsáveis pela arrecadação acima de 1 bilhão de dólares, 9 apresentam o grupo amida e 3, o grupo sulfonamida. ${ }^{5}$

Tendo em vista sua importância, as macrolactamas vêm sendo amplamente estudadas sob vários aspectos: isolamento e elucidação estrutural de produtos naturais, síntese, modificações moleculares, estudos de relação estrutura química/atividade biológica, mecanismo de ação e triagem clínica.

\section{MACROLACTAMAS NATURAIS E SEMI-SINTÉTICAS COM ATIVIDADE BIOLÓGICA}

Diversos depsipeptídeos macrocíclicos apresentam atividade biológica: criptoficina A (atividade antitumoral ${ }^{6}$ ), hapalosina (atividade citotóxica leve ${ }^{7}$ ) e estevastelina $\mathrm{B}$ (inibe ativação de células T e $\mathrm{B}^{8}$ ) (Esquema 1). Outros depsipeptídeos cíclicos, isolados de cianobactéria marinha (Lyngbya majuscula) e denominados lingbiabelinas A e B (Esquema 1), apresentam atividade contra células tumorais. ${ }^{9,10}$

A indolactama-V e benzolactama-V8 (Esquema 2), de 9 e 8 membros respectivamente, ligam-se à proteína quinase $\mathrm{C}$ ativando-a para a promoção de tumores. Esta atividade parece estar diretamente

*e-mail: pradora@farmacia.ufmg.br

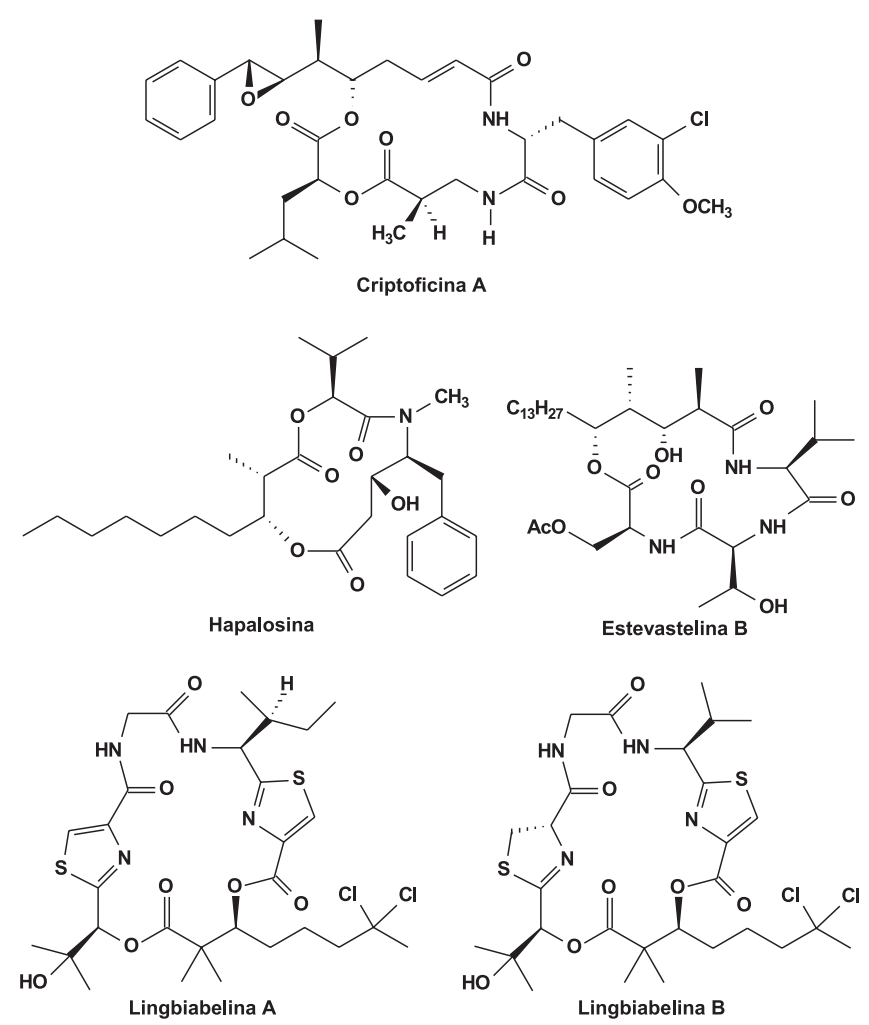

Esquema 1. Depsipeptídeos com atividade biológica<smiles>CC(C)[C@H](C(=O)NC(CO)Cc1c[nH]c2cccc(N)c12)N(C)C</smiles>

(-) Indolactama-V<smiles>CC(C)[C@H]1C(=O)NC(CO)Cc2ccccc2N1C</smiles>

Benzolactama-V8
Esquema 2. Indolactama-V e Benzolactama-V8 


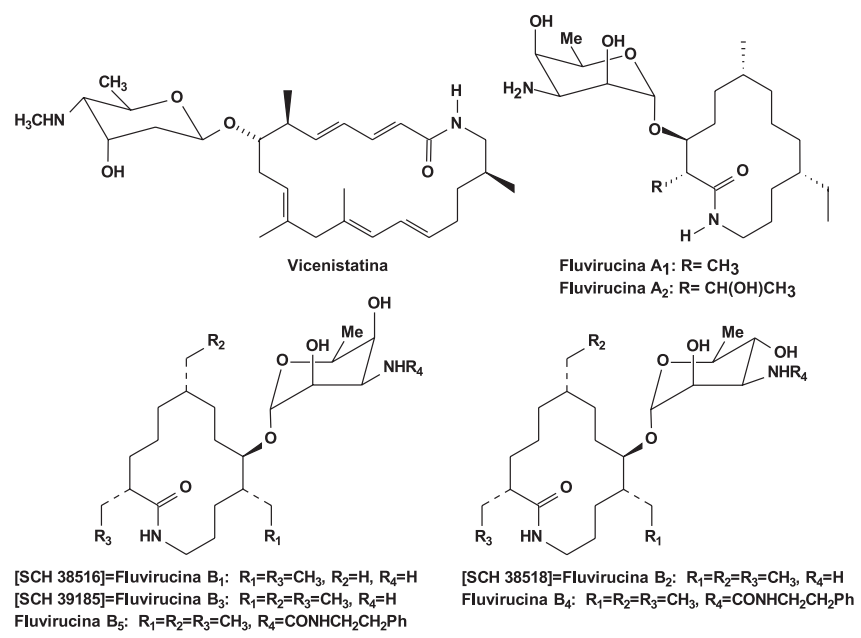

Esquema 3. Macrolactamas ligadas a açúcares
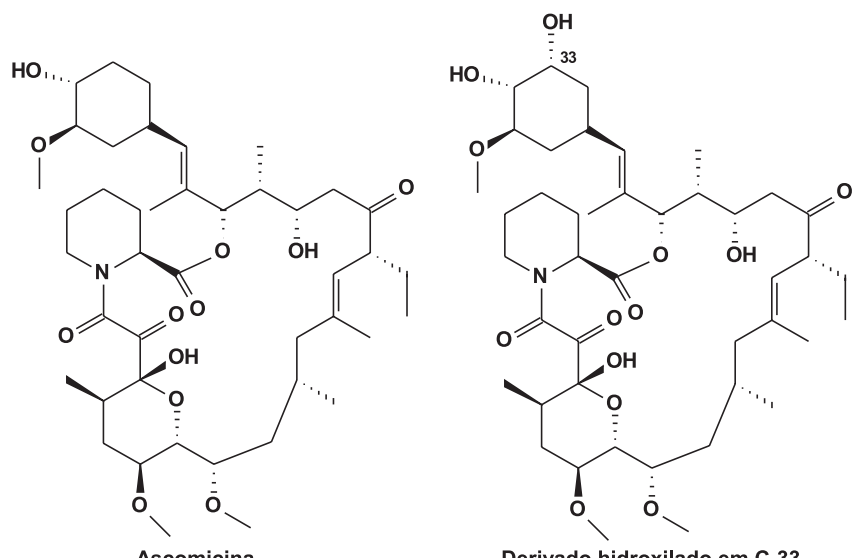

Esquema 4. Ascomicina e seu derivado hidroxilado em C-33

relacionada à presença do hidrogênio da amida, uma vez que lactonas análogas não exibiram esta atividade. ${ }^{11}$

A vicenistatina (Esquema 3), uma macrolactama de 20 membros ligada a um aminoaçúcar e produzida por Streptomyces halstedii HC34, apresenta atividade antitumoral. ${ }^{12}$ As fluvirucinas (Esquema 3), outros glicosídeos macrolactâmicos, foram isoladas como produtos da fermentação de Actinomadura sp e apresentam atividade contra influenza A (fluvirucinas $\mathrm{A}_{1}, \mathrm{~A}_{2}, \mathrm{~B}_{1}-\mathrm{B}_{5}$ ), ${ }^{13,14}$ antifúngica contra $C a n$ dida ssp (fluvirucinas $\left.\mathrm{B}_{1}-\mathrm{B}_{3}\right)^{13,14}$ e contra dermatófitos (fluvirucinas $\left.\mathrm{B}_{1}-\mathrm{B}_{3}\right) \cdot{ }^{15-18}$

A ascomicina (Esquema 4) é um produto de fermentação de Streptomyces hygroscopicus com atividade antifúngica e imunomoduladora, ${ }^{19,20}$ mas com baixa solubilidade em meio aquoso. Kawai e colaboradores ${ }^{21}$ sintetizaram um derivado hidroxilado na posição 33 (Esquema 4) que manteve a atividade imunossupressora da ascomicina in vivo e in vitro e é 100 vezes mais solúvel em meio aquoso em pH 6,5 e 300 vezes mais solúvel em $\mathrm{pH} 7,4 .^{21}$

Macrolactamas com atividade imunomoduladora (Esquema 5) tornaram-se alternativas aos glicocorticóides para o tratamento tópico de doenças inflamatórias crônicas de pele e à ciclosporina para profilaxia e tratamento de rejeição em pacientes submetidos a transplantes de fígado e rim. ${ }^{22} \mathrm{O}$ tacrolimus (FK506), um análogo da ascomicina, foi isolado de uma cultura de Streptomyces tsukubaensis e apresenta atividade imunossupressora semelhante à da ciclosporina in vivo e in vitro. É altamente hidrofóbico e, assim como a ciclosporina, atua inibindo a ativação e maturação de células T. O tacrolimus é usado
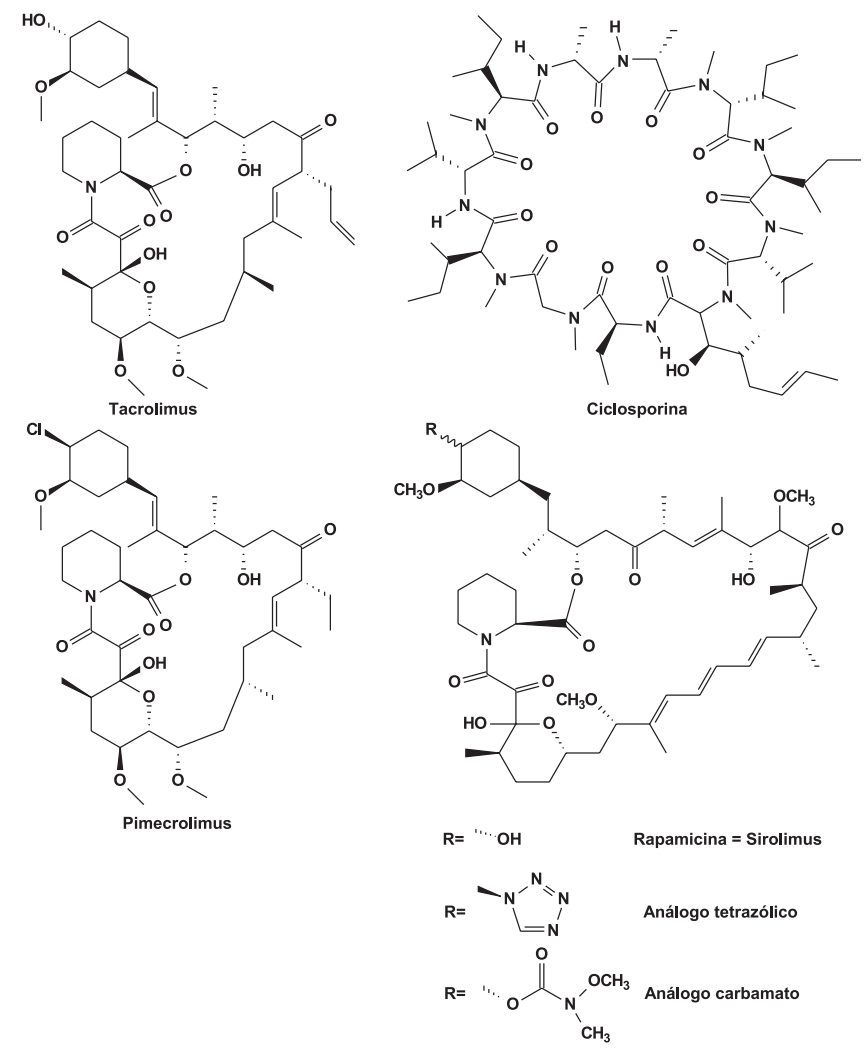

Esquema 5. Macrolactamas com atividade imunomoduladora

por via oral para evitar e tratar rejeição de transplantes e tratar várias doenças da pele, além de ser usado topicamente, na forma de pomadas, no tratamento de dermatite atópica. ${ }^{22,23} \mathrm{O}$ pimecrolimus (32-epicloroascomicina, SDZ ASM 981, Elidel $^{\circledR}$ ) é de uso tópico e seus efeitos são semelhantes aos do tacrolimus. ${ }^{20,22}$ Outra macrolactama imunomoduladora é o sirolimus ou rapamicina, um metabólito secundário fúngico, usado por via oral para prevenção de rejeição no caso de transplante de fígado. ${ }^{22,24} \mathrm{~A}$ rapamicina apresenta tempo de meia-vida longo (63 h), o que acarreta efeitos tóxicos quando o uso é prolongado. Em 2005, Wagner e colaboradores ${ }^{24}$ relataram a síntese de análogos tetrazólico e carbamato da rapamicina que apresentaram atividade imunossupressora, mas tempo de meia-vida menor que a rapamicina. ${ }^{24}$

A leinamicina (Esquema 6), macrolactama de 18 membros produzida por Streptomyces atroolivaceus, apresenta atividades antimicrobiana e antitumoral contra tumores resistentes a outros fármacos. ${ }^{25,26} \mathrm{Um}$ trabalho publicado recentemente sobre síntese e avaliação da citotoxidade de derivados simplificados da leinamicina demonstra a importância desta classe de substâncias. ${ }^{27}$

Trieno-ansamicinas antitumorais (TCM-135 A e B, Esquema 6) foram isoladas de cepas de Streptomyces $\mathrm{sp}^{28}$ Outras ansamicinas antitumorais são a geldanamicina, que possui derivados em triagem clínica, a trienomicina A e a reblastatina ${ }^{29-32}$ (Esquema 6).

Três macrolactamas (ripromicina, ikarugamicina e um epóxido da ikarugamicina, Esquema 7) isoladas entre os produtos de fermentação de cepas de Tü 6239, uma nova espécie de Streptomyces, apresentam atividade contra bactérias Gram positivas e efeito citostático sobre células tumorais humanas. ${ }^{33}$

A maitansina (Esquema 8), isolada de cascas de Maytenus ovatus ${ }^{34}$ apresenta atividade antimitótica superior à da vincristina e vimblastina em testes in vitro, mas demonstrou baixo índice terapêutico. ${ }^{35,36} \mathrm{~A}$ maitansina tornou-se um protótipo para a síntese de antitumorais mais potentes, como aquele com o grupo metilditio- 


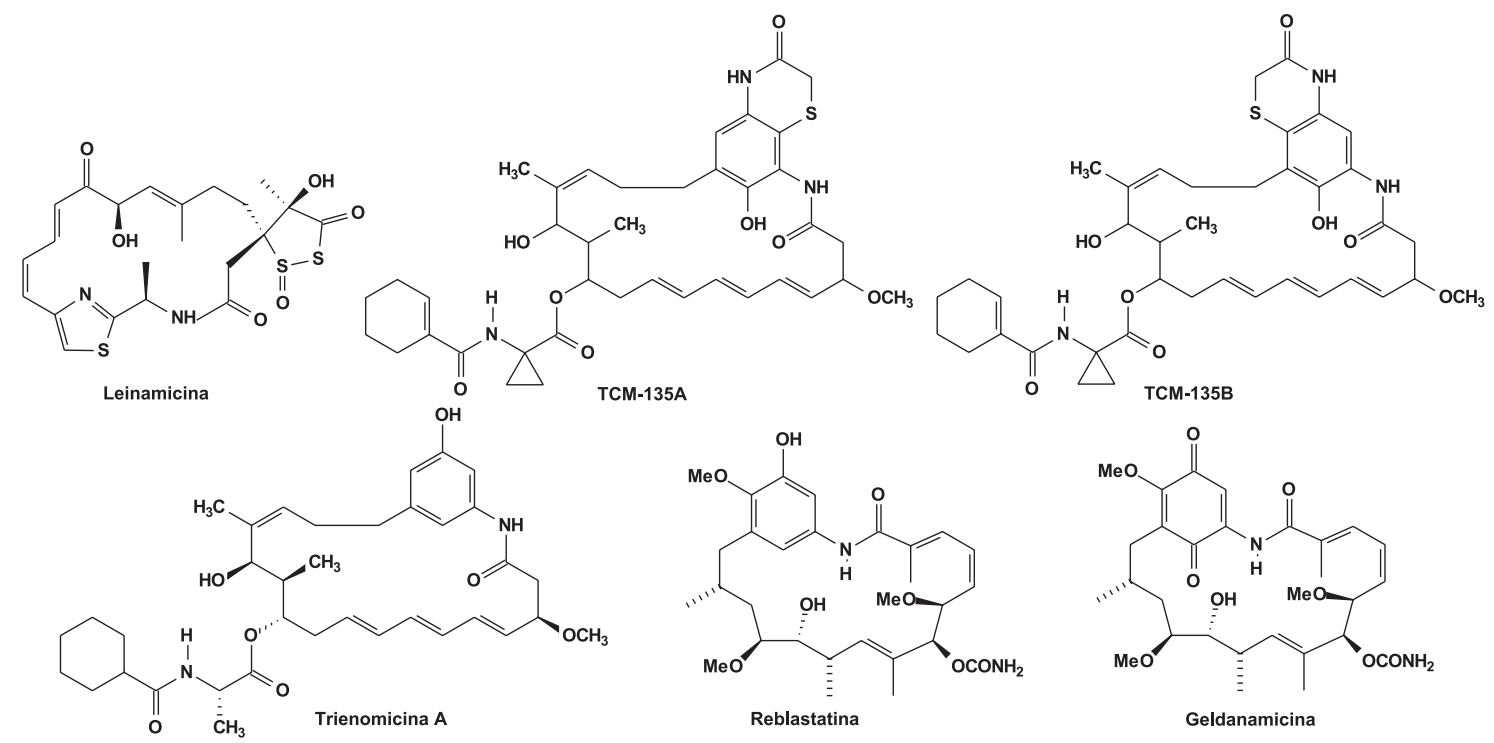

Esquema 6. Leinamicina e ansamicinas

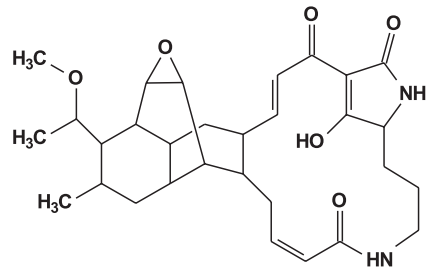

Ripromicina

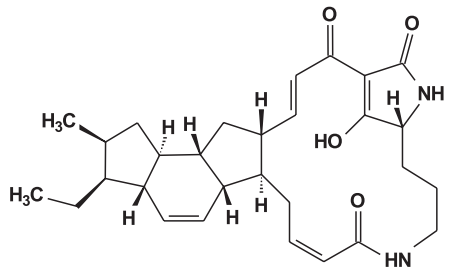

Ikarugamicina

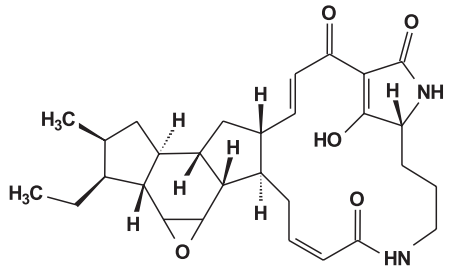

Epóxido da ikarugamicina

Esquema 7. Macrolactamas isoladas de cepas de Tü6239

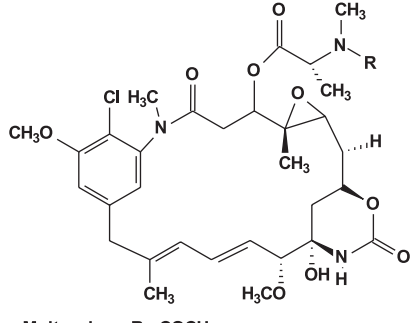

Maitansina: $\mathrm{R}=\mathrm{COCH}_{3}$ Derivado da Maitansina: $\mathrm{R}=\mathrm{COCH}_{2} \mathrm{CH}_{2} \mathrm{SSCH}_{3}$

Esquema 8. Maitansina, seu derivado metilditiopropanoíla e mixovirescina A 1

propanoíla (Esquema 8) em substituição ao grupo acetila presente na maitansina. ${ }^{36}$

As mixovirescinas são macrolactamas de 28 membros isoladas da fermentação de Myxococcus virescin cujo principal componente é a mixovirescina $\mathrm{A}_{1}$ (Esquema 8), que inibe o crescimento de $E$. coli e de outras enterobactérias..$^{37,38}$

O composto K-13 (Esquema 9), produzido por Micromonospora halophytica, é um potente inibidor da ECA com atividade anti-hipertensiva. Outro dímero de tirosina, o OF-4949-I (Esquema 9) produzido pelo fungo Penicilium rugulosum, é inibidor da aminopeptidase B. A bastadina-5 (Esquema 9), produzida por Ianthella basta, bloqueia canais de cálcio do retículo sarcoplasmático e é usada no tratamento de enfermidades relacionadas a músculos esquelético e cardíaco. ${ }^{39-41}$

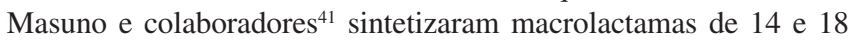
membros, análogos da bastadina-5, que são potenciais agentes para o<smiles>C[C@@H](N)C(=O)NC(Cc1ccc(O)cc1)C(=O)N[C@@H](Cc1ccc(O)c(Oc2ccc(CN(C)C(=O)O)cc2)c1)C(=O)O</smiles><smiles>NCC(=O)N[C@@H]1C(=O)NC(C(=O)O)Cc2ccc(cc2)Oc2cc(ccc2O)OC(=O)C1O</smiles><smiles>CC(C)(C)c1ccc(Oc2cc(CCNC(=O)/C(Cc3cc(Br)c(O)c(Oc4cc(C/C(=N/O)C(=O)NCCc5cc(Br)c(O)c(Oc6cc(Br)cc(Br)c6O)c5)cc(Br)c4O)c3)=N/O)cc(Br)c2Br)c(Br)c1</smiles>

Esquema 9. Macrolactamas contendo dímero de tirosina

tratamento de arritmias e falhas cardíacas. ${ }^{41}$

Ustiloxinas e fomopsinas (Esquema 10), isoladas de culturas de Ustilaginoidia virens e de Phomopsis leptostromiformis, respectivamente, são macrolactamas de 13 membros com atividade antimitóticas, que se ligam à tubulina e interferem na função microtubular celular. ${ }^{42-44}$ 


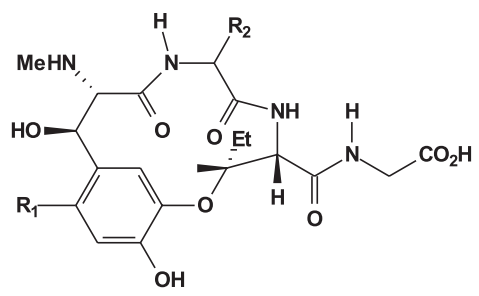

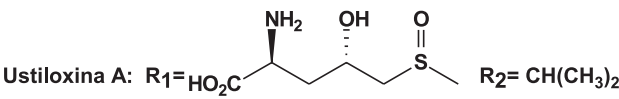

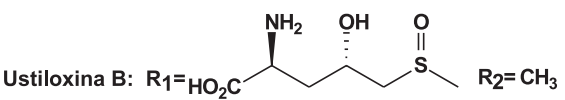<smiles>O=S([18F])CCO</smiles>

Ustiloxina D: $\mathrm{R}_{\mathbf{1}}=\mathrm{H} \mathrm{R}_{\mathbf{2}}=\mathrm{CH}\left(\mathrm{CH}_{3}\right)_{2}$ Ustiloxina $\mathrm{F}: \mathrm{R}_{\mathbf{1}}=\mathrm{H} \quad \mathrm{R}_{\mathbf{2}}=\mathrm{CH}_{3}$

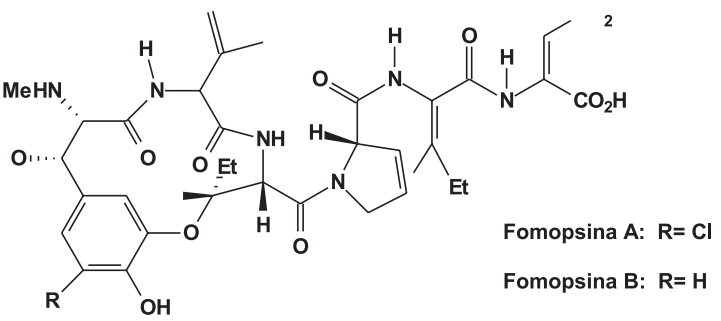

Esquema 10. Ustiloxinas e fomopsinas

\section{MACROLACTAMAS SINTÉTICAS COM ATIVIDADE BIOLÓGICA}

Embora a síntese de macrociclos seja considerada um dos desafios da síntese orgânica, diversos grupos de pesquisa têm se dedicado à síntese desses potenciais agentes bioativos, ${ }^{1}$ inclusive de macrolactamas.

Lactamização em substratos aminoácidos levou às macrolactamas 1, 2 e 3 (Esquema 11) e todas apresentaram moderada atividade citotóxica em testes in vitro. ${ }^{45}$

Macrociclos taxóides, derivados conformacionalmente restritos do paclitaxel, foram sintetizados pelo método de fechamento de anel por metátese de olefina ( $R C M$ - ring-closing metathesis) com rendimentos entre 70 e $90 \%{ }^{46}$ Entre os produtos sintetizados, as macrolactamas 4 e 5 (Esquema 12) destacaram-se quanto à atividade citotóxica. ${ }^{46}$

Jefferson e colaboradore ${ }^{47}$ sintetizaram e avaliaram a atividade antibacteriana de uma série de "conjugados quinolona-macrociclo". A formação dos macrociclos envolveu reação de substituição nucleofílica aromática em presença de base (Esquema 13). Os conjugados 6 e 7, derivados do ácido nalidíxico, foram menos potentes que a quinolona de origem e os conjugados $\mathbf{8}$ e $\mathbf{9}$, derivados da ciprofloxacina e da ofloxacina, respectivamente, apresentaram atividade contra $E$. coli $\mathrm{e} S$. aureus semelhante às das quinolonas. A substância 10, análogo de cadeia aberta de 9, foi inativa, indicando que 9 não é um pró-fármaco da ofloxacina e que a presença da unidade macrocíclica, conformacionalmente restrita, é importante para a atividade..$^{47}$

Na síntese das macrolactamas 11-15 (Esquema 14) utilizaram-se aminoésteres (etílico e metílico) como substratos e trietoxiantimônio ou boronamida como catalisadores na etapa de lactamização. ${ }^{48}$ As

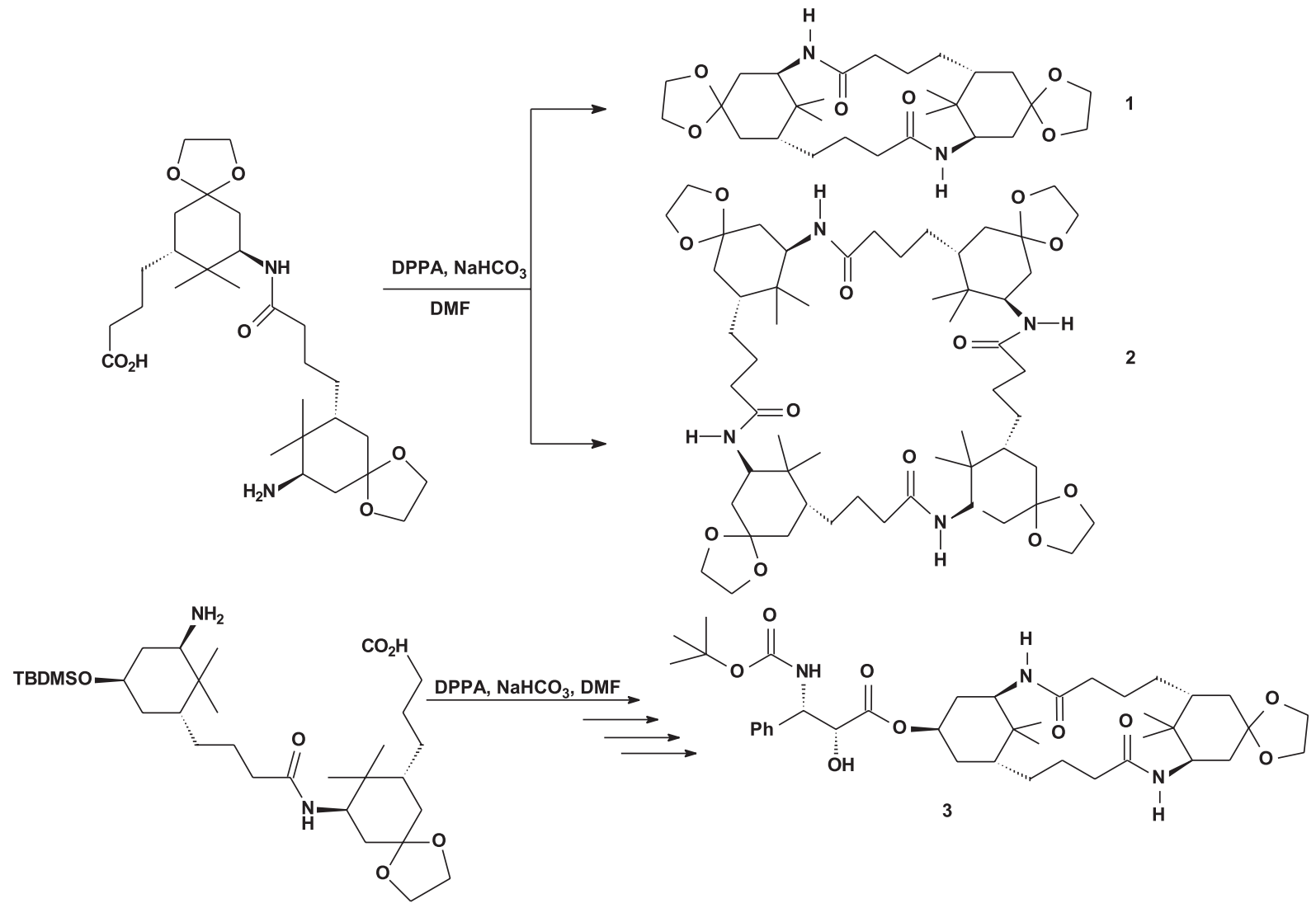

Esquema 11. Síntese de macrolactamas citotóxicas 


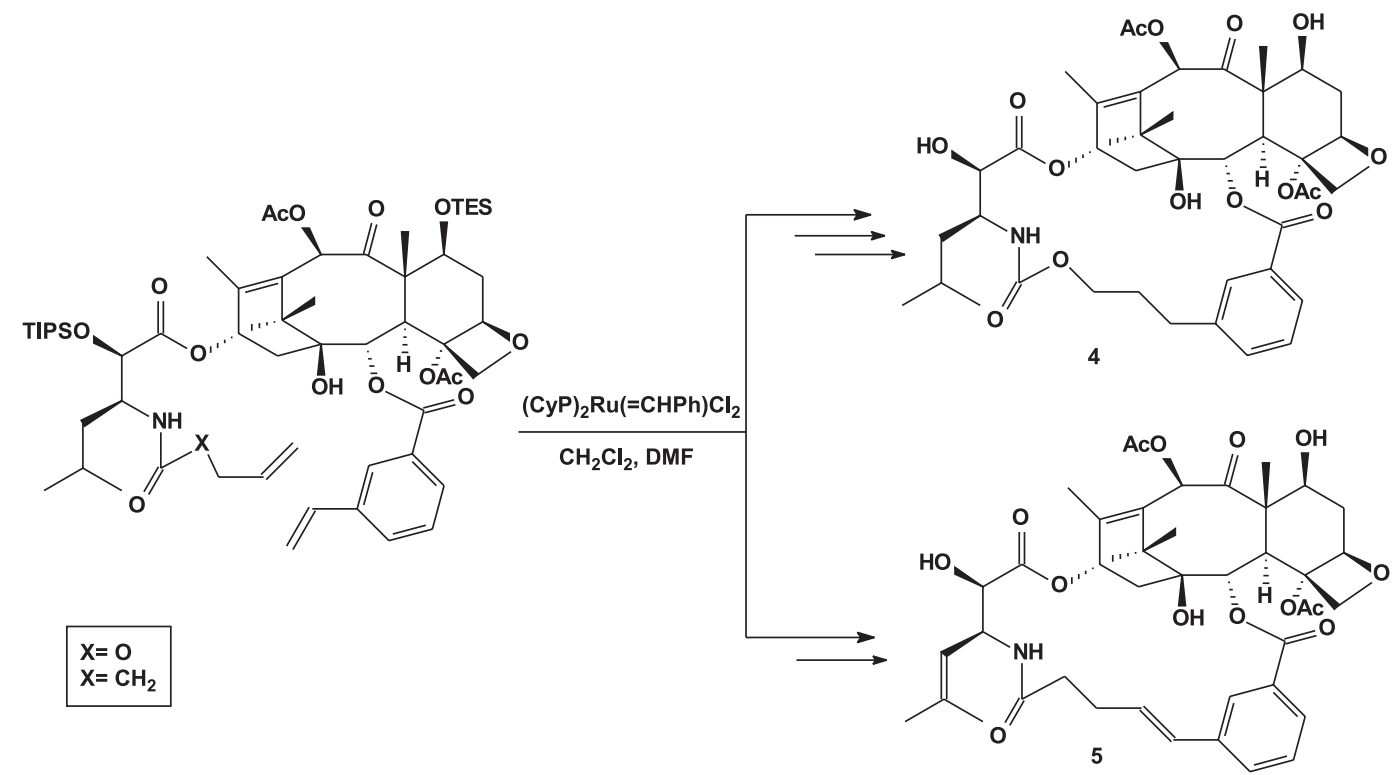

Esquema 12. Síntese de macrociclos taxóides
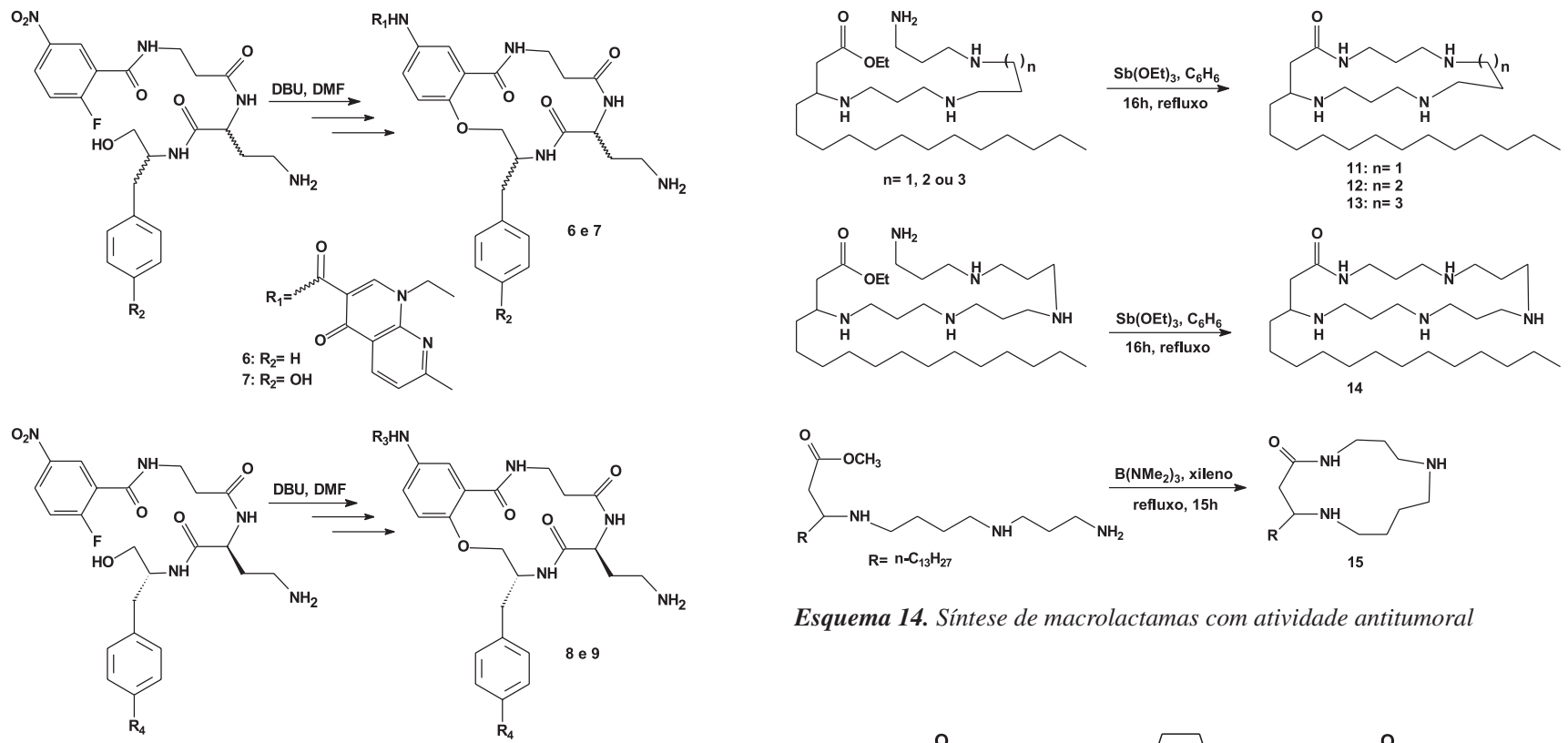

Esquema 14. Síntese de macrolactamas com atividade antitumoral
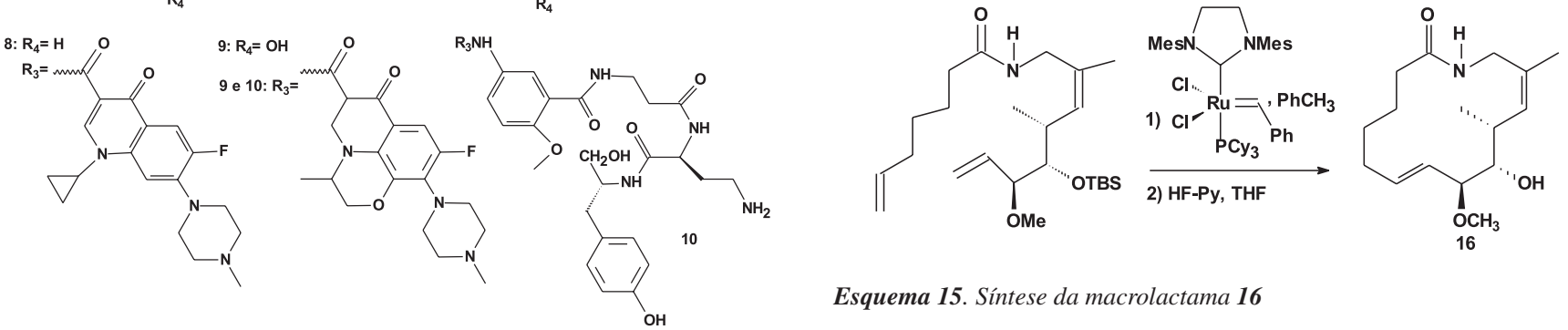

Esquema 15. Síntese da macrolactama 16

Esquema 13. Síntese de "conjugados quinolona-macrociclo"

macrolactamas inibiram o crescimento de células tumorais humanas de próstata (DuPro e PC-3) em testes in vitro, além de causar depleção de ATP nas células tumorais, o que pode explicar o efeito citotóxico destes compostos. ${ }^{48}$

Para a síntese da macrolactama $\mathbf{1 6}$ (Esquema 15), que apresentou potente inibição de metástase em testes in vitro,${ }^{49}$ foi utilizada reação

de metátese de alqueno na etapa de ciclização (60\%), seguida de remoção do grupo protetor $(81 \%){ }^{50,51}$

Stachel e colaboradores ${ }^{4}$ relataram que a macrolactama de 15 membros 18, com restrição conformacional em relação a seu análogo 17, é um inibidor mais potente de $\beta$-secretase (alvo atrativo na busca de agentes para o tratamento da Doença de Alzheimer - DA) que 17 (Esquema 16). Entre as macrolactamas de 15 e 14 membros (19-23, Esquema 16), 23 destacou-se pela potente atividade inibidora de $\beta$-secretase in vitro e redução da formação de amilóide (peptídeo 

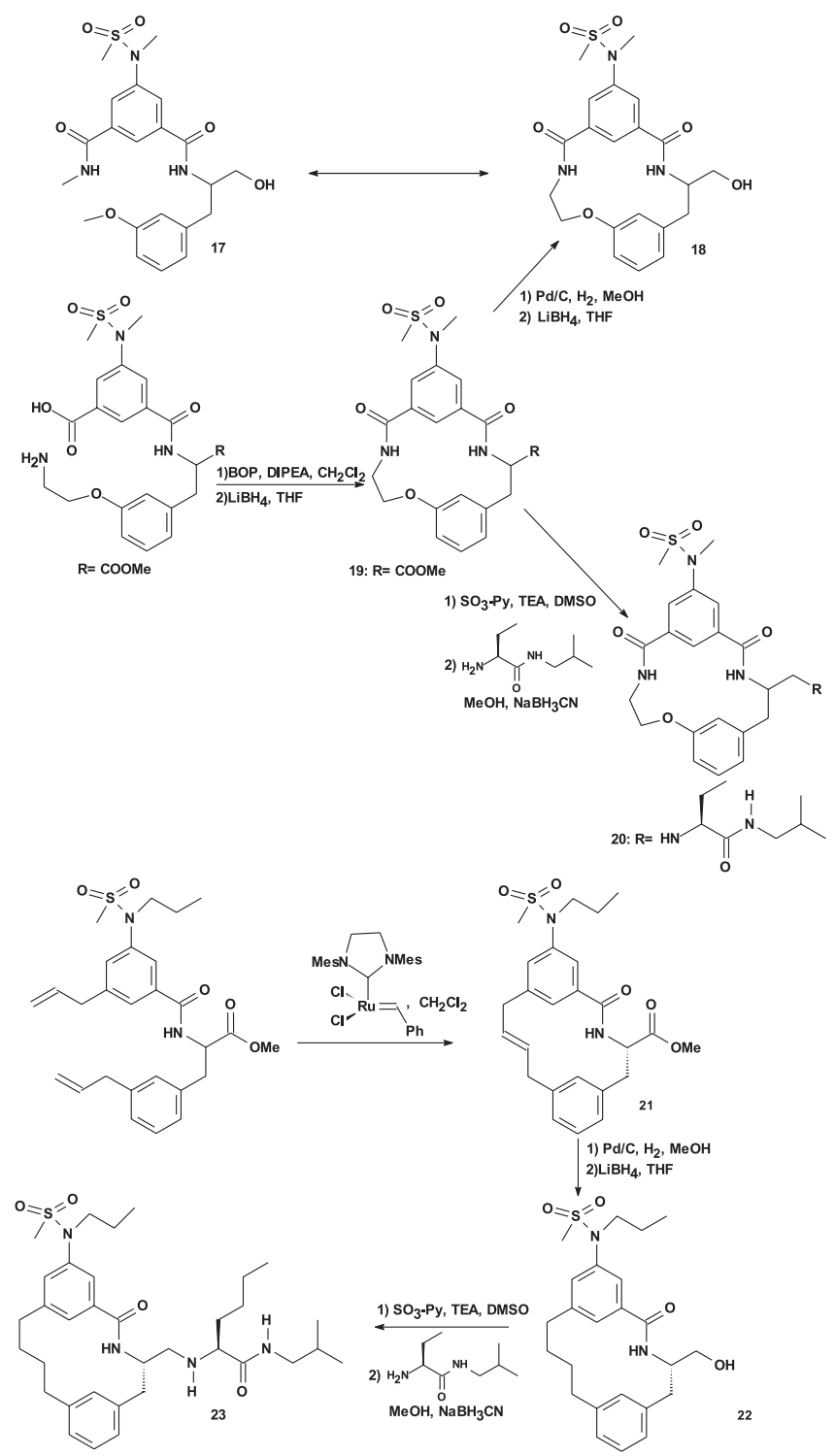

Esquema 16. Sintese de inibidores de $\beta$-secretase

neurotóxico associado à DA) in vivo. ${ }^{4} \mathrm{~A}$ reação de ciclização para a obtenção de 19, que, posteriormente, foi transformada em $\mathbf{1 8}$ e $\mathbf{2 0}$, envolveu lactamização. A macrolactama 21, precursora de 22 e 23, foi sintetizada por metátese de olefina ${ }^{4}$ (Esquema 16).

\section{SÍNTESE DE MACROCICLOS POR REAÇÃO DE CARBO- CICLIZAÇÃO RADICALAR MEDIADA POR HIDRETO DE TRI- $N$-BUTILESTANHO}

Diferentes métodos têm sido empregados para a obtenção de macrociclos, como o de carbociclização radicalar mediada por hidreto de tri- $n$-butilestanho. ${ }^{52,53}$ As principais características das reações com $\mathrm{Bu}_{3} \mathrm{SnH}$, responsáveis pela sua ampla aplicação, são a relativa simplicidade do ponto de vista operacional; a ciclização ocorre sem alteração da configuração dos estereocentros de precursores quirais; a maioria dos grupos funcionais é inerte ao reagente. ${ }^{54,55}$ No entanto, há inconvenientes: toxicidade, dificuldade em eliminar os resíduos de estanho dos produtos obtidos e formação de produtos acíclicos reduzidos. ${ }^{56-58}$

Os mecanismos envolvidos nas reações com $\mathrm{Bu}_{3} \mathrm{SnH}$ em presença de um iniciador radicalar encontram-se bem discutidos ${ }^{59-64}$ e estão representados para o 7-iodo-2-hepteno no Esquema 17. O radical $\mathrm{Bu}_{3} \mathrm{Sn}$. abstrai o átomo de halogênio do substrato e o novo intermediário radicalar formado é capaz de: ciclizar pelo modo endo e/ou exo, levando a um novo radical ciclizado, que é reduzido pelo $\mathrm{Bu}_{3} \mathrm{SnH}$; abstrair um hidrogênio do $\mathrm{Bu}_{3} \mathrm{SnH}$, levando a um produto acíclico reduzido (hidrogenólise); abstrair um hidrogênio do próprio substrato (transferência 1,5 ou 1,6), alterando a posição do radical, que pode ciclizar ou abstrair um hidrogênio do $\mathrm{Bu}_{3} \mathrm{SnH}$ para levar ao produto de redução. O caminho seguido depende das constantes de transferência de hidrogênio e de ciclização das espécies envolvidas, além da concentração do substrato e do $\mathrm{Bu}_{3} \mathrm{SnH}$.
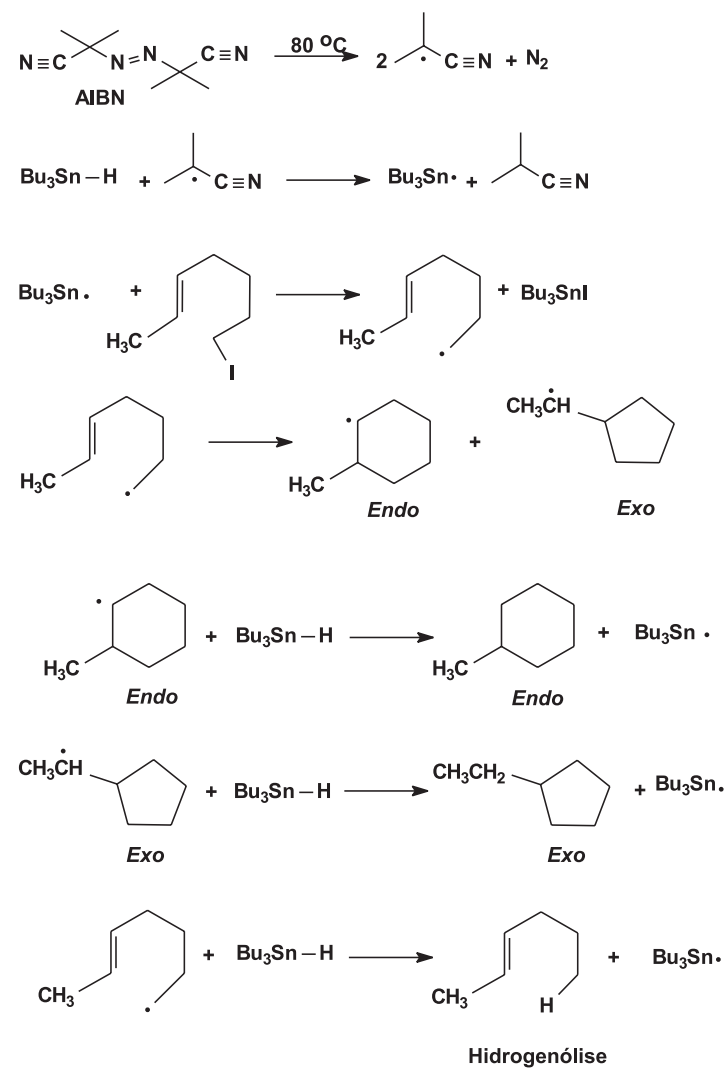

Esquema 17. Mecanismos envolvidos nas reações com Bu $\mathrm{S}_{3} \mathrm{SH} / \mathrm{AIBN}$

Embora os estudos sobre macrociclização radicalar com $\mathrm{Bu}_{3} \mathrm{SnH}$ sejam restritos quando comparados com aqueles de obtenção de ciclos de cinco e seis membros, ${ }^{65}$ já é de conhecimento que macrociclos podem ser obtidos com bons rendimentos se precursores adequados forem utilizados e se certas condições de reação forem observadas.

Os estudos pioneiros desenvolvidos por Porter e colaboradores ${ }^{66-68}$ sobre macrociclização radicalar envolvendo ataque de radicais alquila a ligações $\mathrm{C}=\mathrm{C}$ permitiram estabelecer os seguintes critérios: a ciclização é favorecida nos substratos em que a ligação dupla se encontra ativada por um grupo retirador de elétrons; alta diluição do substrato ( 3 a $7 \mathrm{mmol} / \mathrm{L}$ ) melhora o rendimento de produtos macrocíclicos, uma vez que diminui a probabilidade da ocorrência de processos bimoleculares, como redução direta do radical e reações intermoleculares; em geral, precursores iodados conduzem a melhores rendimentos de produtos ciclizados que precursores bromados; em geral, as macrociclizações endo são preferenciais em relação às exo.

Porter e colaboradores ${ }^{66}$ relataram a obtenção de macrociclos de 10,14 e 18 membros provenientes de ciclização pelo modo endo com rendimentos de 15, 63 e 54\%, respectivamente, por reação de $\omega$-iodoenonas com $\mathrm{Bu}_{3} \mathrm{SnH} / \mathrm{AIBN}$ (Esquema 18). 


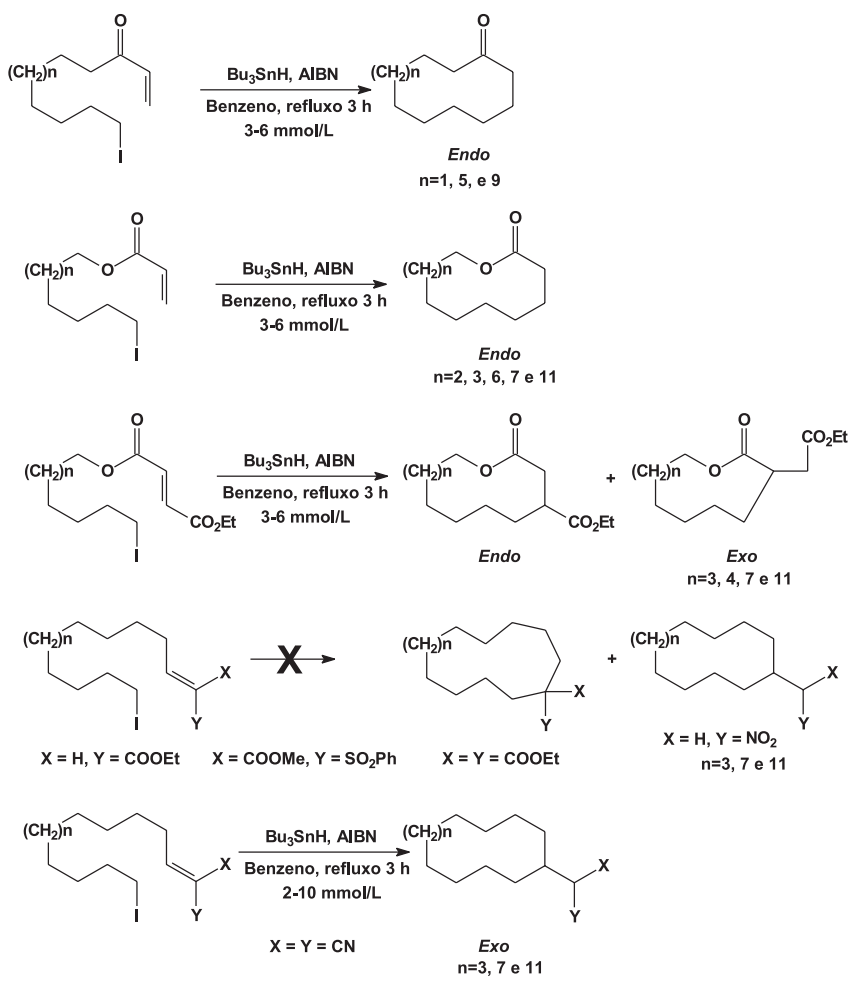

Esquema 18. Macrociclos obtidos por Porter e colaboradores

Porter e Chang ${ }^{67}$ obtiveram macrolactonas de 11, 12, 15, 16 e 20 membros (Esquema 18), também formadas pelo modo endo, a partir de ésteres acrilatos e os macrociclos de 15, 16 e 20 membros foram obtidos com melhores rendimentos, entre 45 e $56 \%$. A partir de iodofumaratos foram formados macrociclos pelos modos endo (anéis de 12, 13, 16 e 20 membros) e exo, com predominância do modo endo. ${ }^{67}$

A partir de substratos em que o ataque exo seria favorecido eletronicamente, Porter e colaboradores ${ }^{68}$ não obtiveram produtos de ciclização (Esquema 18). Somente precursores altamente ativados por dois substituintes ciano levaram a produtos de 12,16 e 20 membros $\left(<5,35\right.$ e $50 \%$, respectivamente) resultantes de ciclização exo. ${ }^{68}$

Os resultados dos trabalhos de Porter e colaboradores ${ }^{66-68}$ indicam que a adição do radical a alquenos se dá preferencialmente na região menos impedida estericamente, ocorrendo normalmente pelo modo endo. $\mathrm{O}$ modo exo pode ser forçado com a inclusão de dois substituintes altamente retiradores de elétrons $(\mathrm{CN})$ e a ciclização exo tem sua eficácia aumentada em ciclos maiores, o que sugere que a macrociclização exo é estericamente mais exigente que a endo.

Robertson e Hatley ${ }^{69}$ relataram a síntese de um precursor cíclico da roseofilina (25), um antibiótico citotóxico, que foi obtido por reação do derivado iodado 24 com $\mathrm{Bu}_{3} \mathrm{SnH}$ (Esquema 19).

Como parte dos estudos visando a síntese da micotoxina zearalenona, Hitchcock e Pattenden ${ }^{70}$ sintetizaram o E-cetomacrolídeo 27 (14-endo), a partir de 26 (Esquema 20).

Cetonas macrocíclicas de 14 membros de configuração $E$ e $Z$ (Esquema 21) foram obtidas por reação de $\mathbf{2 8} \mathrm{com} \mathrm{Bu}_{3} \mathrm{SnH} / \mathrm{AIBN}$. Por ciclização 14-endo, as duas trienonas formaram-se com $52 \%$ de rendimento e proporção de 3:1 entre os isômeros $E: Z .{ }^{71}$

Robertson e colaboradores ${ }^{72}$ observaram diferenças na ciclização das iodoenonas cis (29) e trans (31) (Esquema 22), sugerindo estereosseletividade para reações de macrociclização radicalar. Além de o rendimento do macrociclo 32, proveniente de 31, ter sido mais baixo, foi preciso maior diluição do substrato. ${ }^{72}$

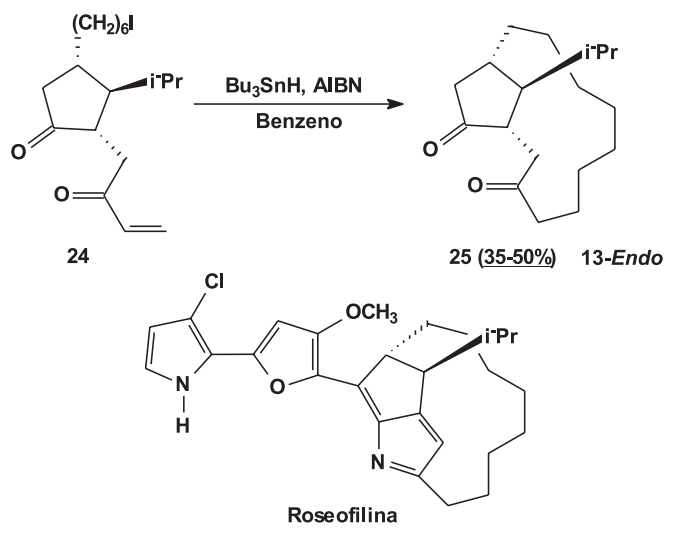

Esquema 19. Síntese de precursor da roseofilina por macrociclização 13 -endo

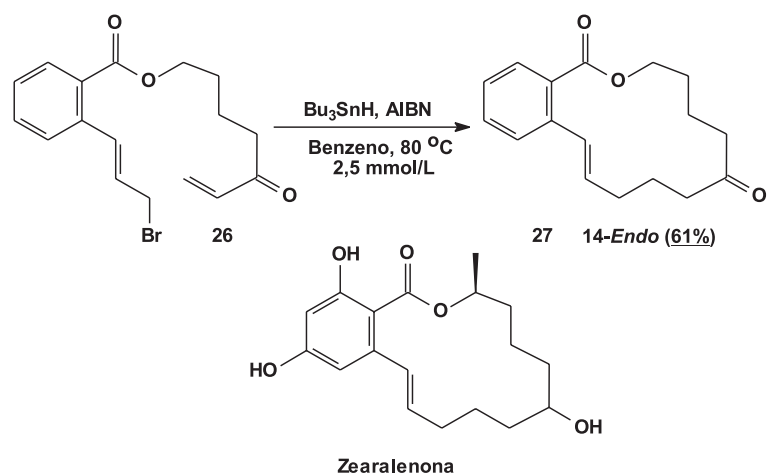

Esquema 20. Síntese de macrolactona precur sora da micotoxina zearalenona

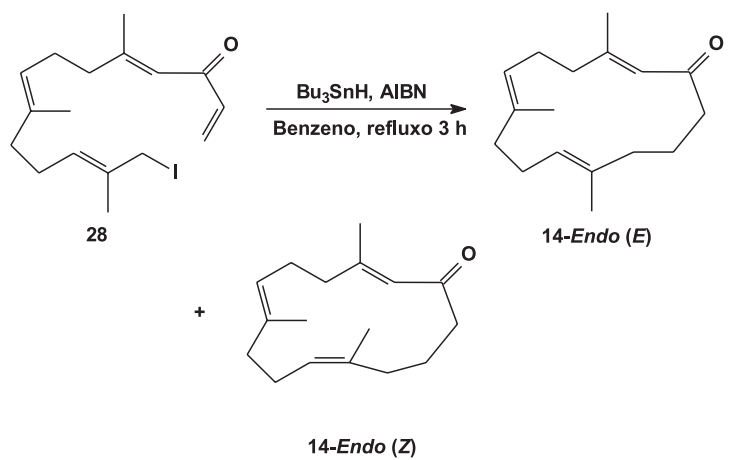

Esquema 21. Síntese de trienonas macrocíclicas de 14 membros
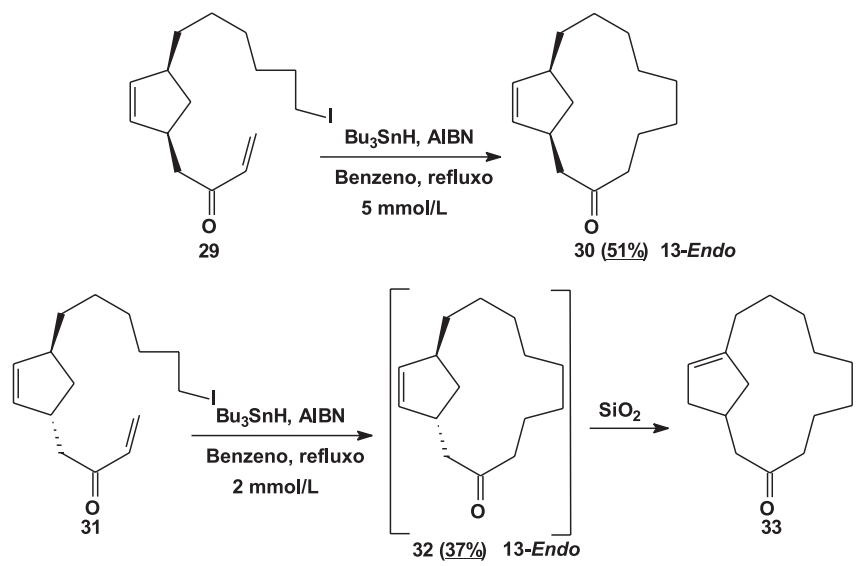

Esquema 22. Macrociclização de iodoenonas cis e trans 
A síntese de macrolactonas e éteres macrocíclicos a partir de $\omega$-iodopolioxaalquilacrilatos e compostos relacionados (Esquema 23) foi relatada por Beckwith e colaboradores. ${ }^{73}$ Os substratos $a-g$ levaram a macrociclos provenientes exclusivamente da ciclização endo. Os precursores $h$ - $k$, com um substituinte que ativa o ataque exo, conduziram a produtos de ciclização endo e exo.

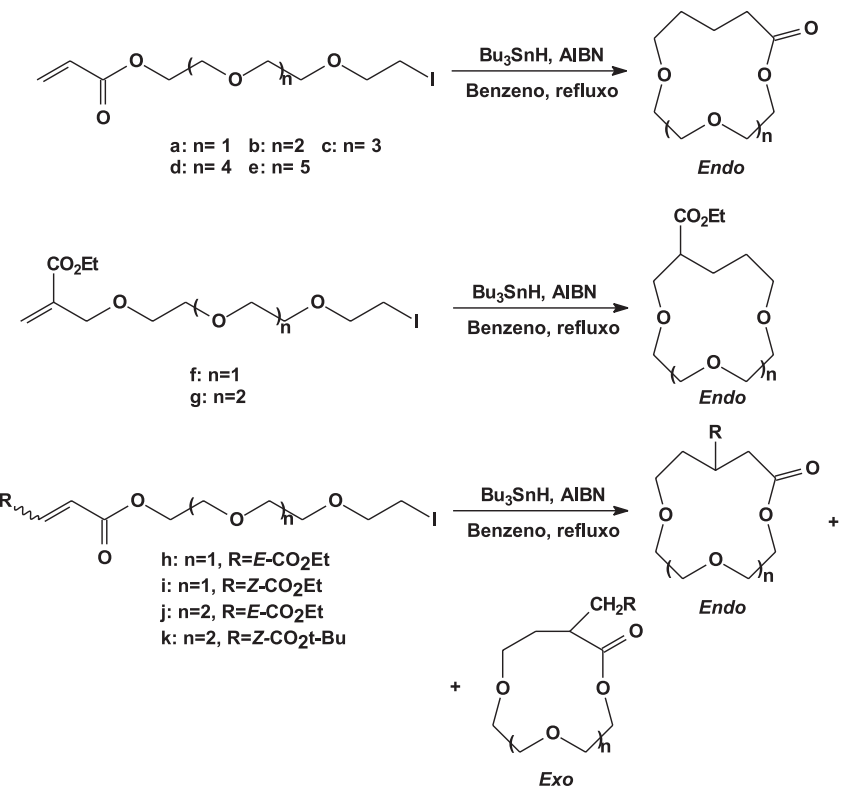

Esquema 23. Síntese de macrolactonas e éteres macrocíclicos por reação radicalar

Reação de macrociclização-transanulação com $\mathrm{Bu}_{3} \mathrm{SnH} / \mathrm{AIBN}$ foi descrita empregando-se o precursor iodotrienona $34^{74}$ (Esquema 24). O radical resultante da ciclização radicalar 13-endo sofreu, em seguida, ciclizações 5 e 6-exo e conduziu ao triciclo 35 . $^{74}$
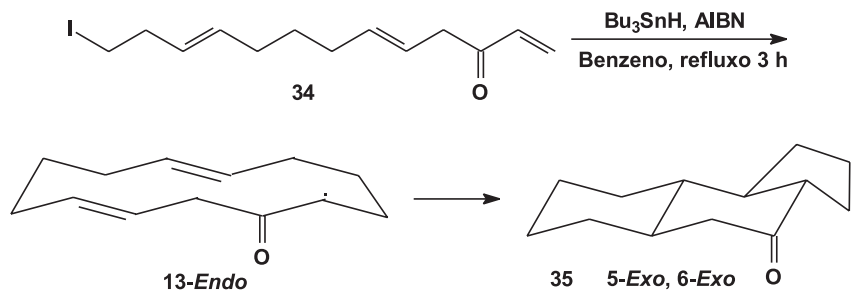

Esquema 24. Ciclização radicalar de iodotrienona

Shea e colaboradores ${ }^{75}$ sintetizaram macrociclos de 22 membros pelo tratamento de biscetais derivados do estireno com $\mathrm{Bu}_{3} \mathrm{SnH}$ (Esquema 25). O radical é gerado por ataque do radical tributilestanila ao carbono mais externo de um dos grupos alquenila e o centro radicalar formado promove ataque endo à ligação dupla da outra unidade do biscetal. ${ }^{75}$

Macrolactonas de 10 a 15 membros foram obtidas pelo modo de ciclização endo (46 a $80 \%$ ) por reação de 2-(tri- $n$-butilestanilmetilpropanoatos) de $\omega$-fenilselenoalquila com $\mathrm{Bu}_{3} \mathrm{SnH}^{76}$ (Esquema 26). O grupo $\mathrm{Bu}_{3} \mathrm{Sn}$ do substrato possibilita a formação do radical $\mathrm{Bu}_{3} \mathrm{Sn}$. in situ pela fragmentação da ligação C-Sn, o que permite usar quantidades catalíticas de $\mathrm{Bu}_{3} \mathrm{SnH}$ e evita a formação de produtos de redução indesejáveis. ${ }^{76}$

Macrociclos têm sido também obtidos por ataque de radicais a grupos acetileno. Lamas e colaboradores ${ }^{77}$ utilizaram a reação radicalar

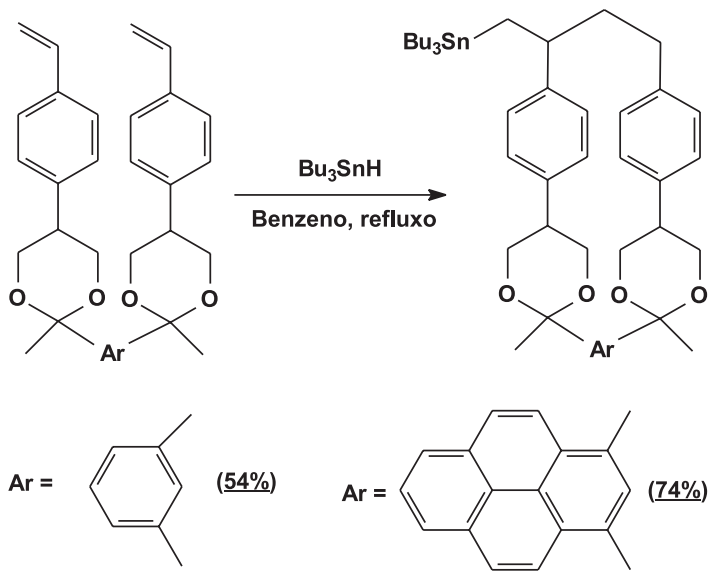

Esquema 25. Síntese de macrociclos de 22 membros

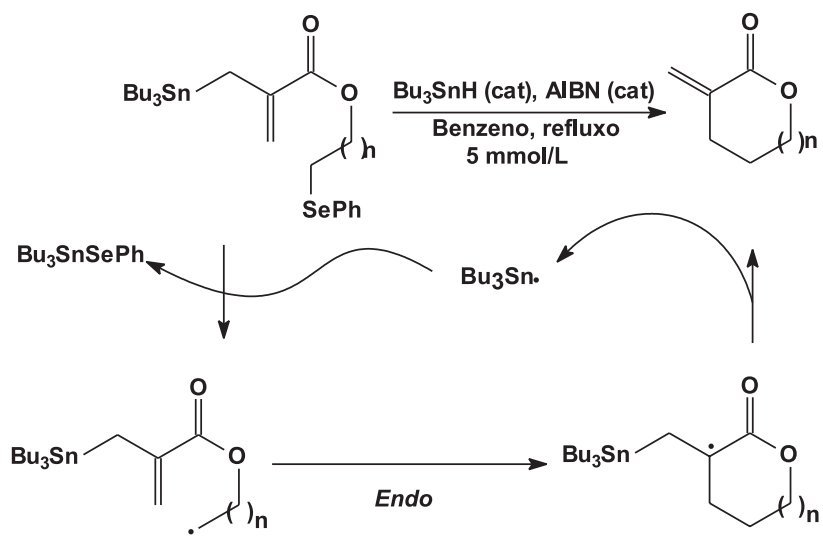

Esquema 26. Obtenção de macrolactonas a partir de 2-(tri-n-butilestanilmetilpropanoatos) de $\omega$-fenilselenoalquila

mediada por $\mathrm{Bu}_{3} \mathrm{SnH}$ para obter precursores do alcalóide lenoxamina a partir dos substratos arilbromoacetilenos 36 e 38 (Esquema 27). Foram obtidas as macrolactamas 10-endo $37(45 \%)$ e 39 (60\%). ${ }^{77}$ No âmbito de estudos realizados para obter precursores do sesquiterpeno periplanona $\mathrm{B}$, Jonas e colaboradores ${ }^{78}$ também obtiveram um macrociclo resultante de ciclização 10-endo (41) (Esquema 27). Como na síntese descrita anteriormente, ${ }^{77}$ o substrato $\mathbf{4 0}$ também apresenta o grupo acetileno como aceptor de radical. ${ }^{78}$ Houldsworth e colaboradores ${ }^{79}$ também utilizaram substrato contendo alquino como eletrófilo. Em estudos visando à síntese de precursores do antitumoral paclitaxel, a iododienona $\mathbf{4 2}$ foi submetida à reação com $\mathrm{Bu}_{3} \mathrm{SnH} / \mathrm{AIBN}$ e o triciclo pentadecadienodiona $\mathbf{4 3}$ foi obtido por uma cascata de ciclizações radicalares: macrociclização 12-endo, seguida de transanulação 6-exo-8-endo ${ }^{79}$ (Esquema 27). Os estereoisômeros cis e trans da macrolactama de 11 membros 45 (Esquema 27) foram obtidos por Rodríguez e colaboradores, ${ }^{80}$ por meio da adição intramolecular de um radical arila, formado a partir do precursor 44, ao grupo trimetilsililacetileno.

Reações de macrociclização com radicais acila foram primeiramente estudadas por Boger e Mathvink. ${ }^{81}$ Substratos $\omega$-fenilselenoésteres acrilatos conduziram a macrociclos de $11,12,14,16$ e 20 membros (Esquema 28), com rendimentos entre 46 e $68 \%$, quando tratados com $\mathrm{Bu}_{3} \mathrm{SnH}$ e AIBN em condições de alta diluição. ${ }^{81}$ Astley e Pattenden ${ }^{82}$ também obtiveram um macrociclo (47) formado por ataque endo do radical acila gerado a partir de $\mathbf{4 6}$ (Esquema 28). O macrociclo 47 apresenta o anel furanocembranóide de 14 membros encontrado na lofotoxina, uma potente substância neurotóxica isolada de corais. ${ }^{82}$ 

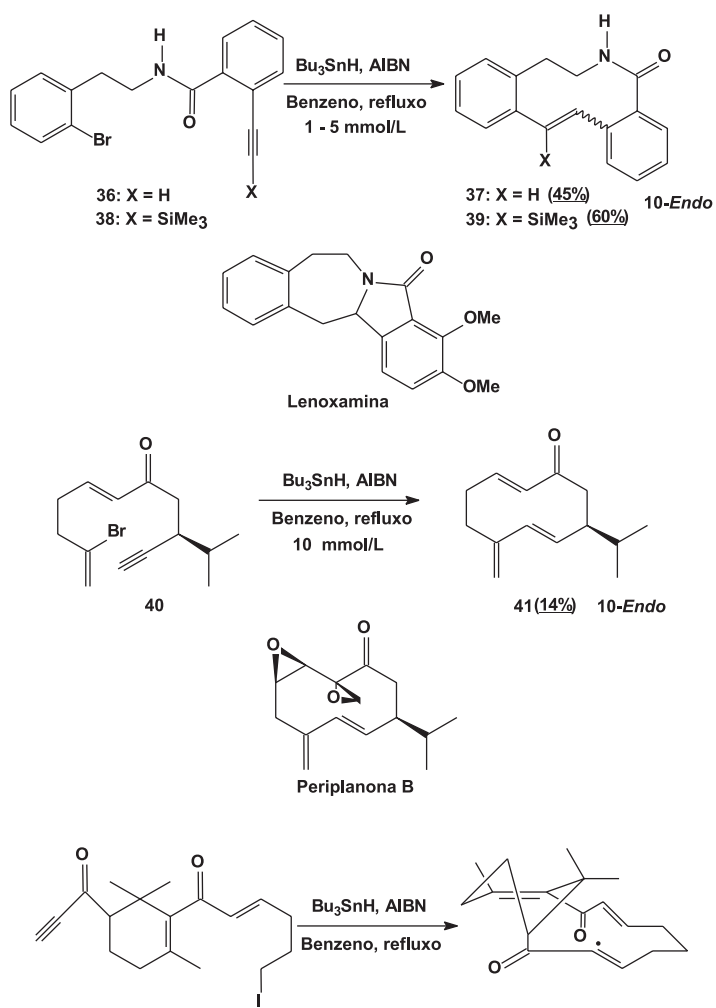

42
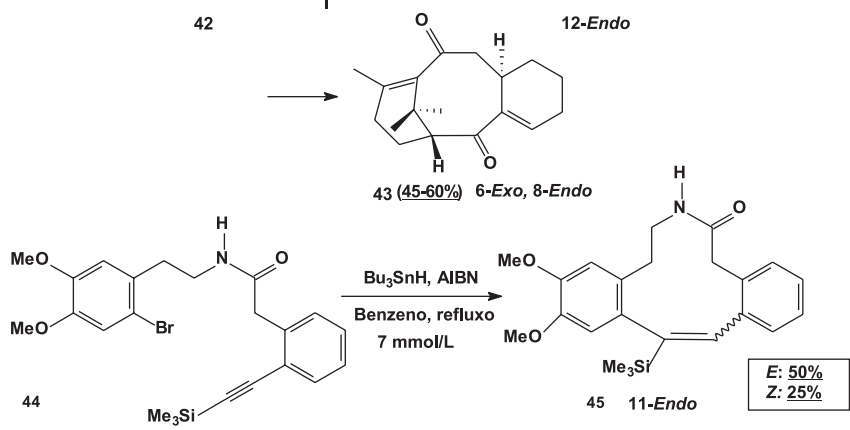

Esquema 27. Síntese de macrociclos a partir de substratos acetilênicos

Ciclizações com $\mathrm{Bu}_{3} \mathrm{SnH}$ envolvendo ataque de radicais arila a um grupo alquenila vêm sendo amplamente utilizadas para a construção de ciclos de cinco e seis membros fundidos a anéis aromáti$\cos ^{83}$ e a partir da década de 90 passaram a ser usadas também para a obtenção de ciclos com mais de seis membros fundidos a anéis benzênicos. ${ }^{52,65,84-87}$

Reação de bromoalil e bromobutenilcicloexanóis 48 e 49 (Esquema 29), respectivamente, $\mathrm{com} \mathrm{Bu}_{3} \mathrm{SnH}$, conduziu a compostos cíclicos fundidos a anéis aromáticos, por meio de ciclização 9-endo de radicais arila. ${ }^{84}$

Por reação de iodobenzenos contendo uma cadeia derivada de aminoácido na posição orto com $\mathrm{Bu}_{3} \mathrm{SnH}$ foram obtidos compostos cíclicos de seis a nove membros, formados pelo modo endo $^{85}$ (Esquema 30).

Nandi e colaboradores ${ }^{86}$ obtiveram ciclos fundidos a anéis aromáticos a partir de derivados da D-glicose (Esquema 31). Nos substratos em que a relação dos substituintes envolvidos na ciclização é cis (50), as ciclizações ocorreram sem dificuldade e os rendimentos dos triciclos variaram entre 50 e $58 \%$. Os produtos de ciclização dos substratos trans $\mathbf{( 5 1 )}$ foram obtidos com rendimentos bem inferiores (15-20\%) e sob condições de alta diluição e adição muito lenta do hidreto de tri- $n$-butilestanho $(8 \mathrm{~h})$. Estes resultados mostram que as
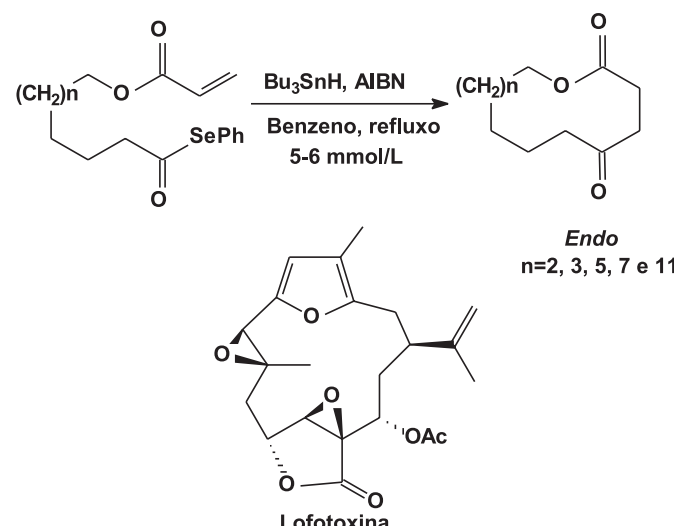

Lofotoxina

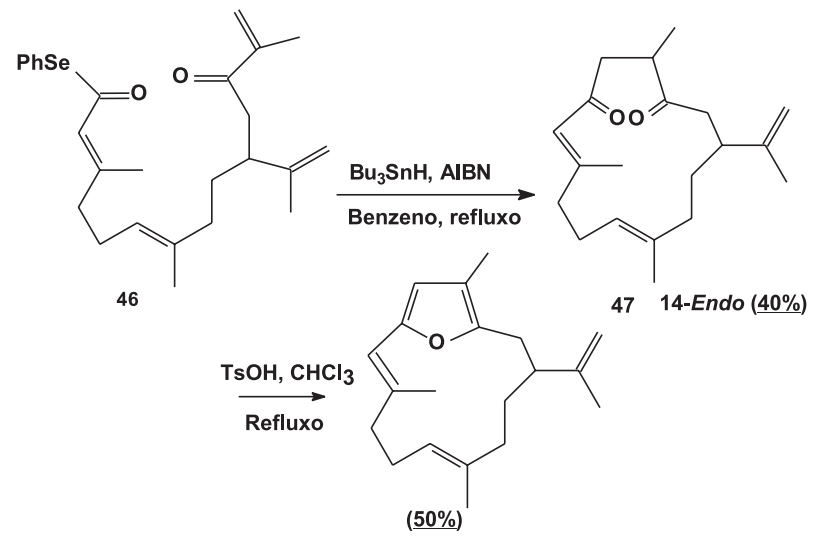

Esquema 28. Macrociclos sintetizados por ataque de radicais acila a grupos alquenila
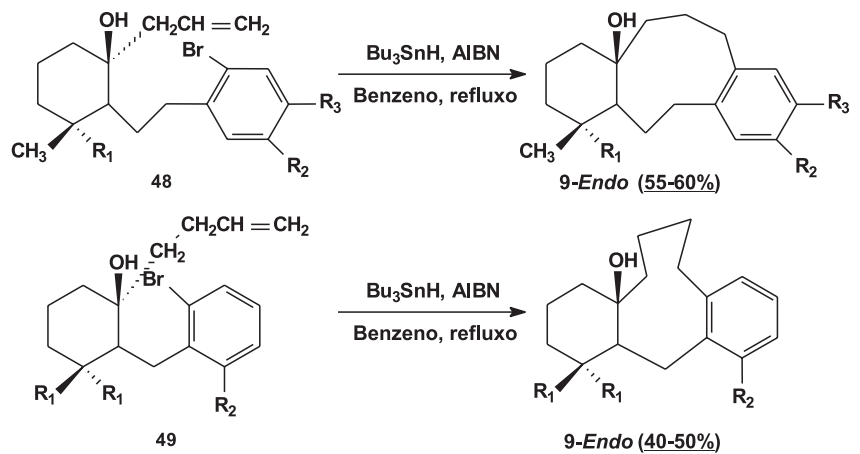

Esquema 29. Ciclizações 9-endo a partir de bromoalil e bromo-butenilcicloexanóis
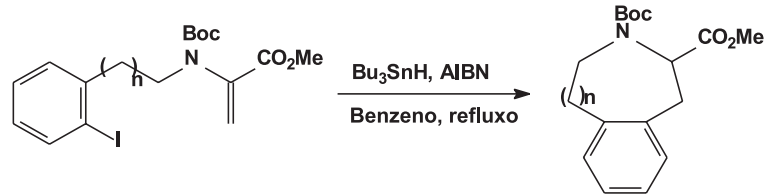

a: $n=0(79 \%)$

b: $n=1(73 \%)$

c: $n=2\left(\frac{71 \%)}{\text { d: } n=3(52 \%)}\right.$

Esquema 30. Sintese de ciclos de seis a nove membros

ciclizações de derivados de carboidratos podem ser estereosseletivas, ${ }^{86}$ o que confirma estudos prévios. ${ }^{72}$ Surpreendentemente, na síntese de triciclos a partir de derivados da D-manose de configuração trans (52) 


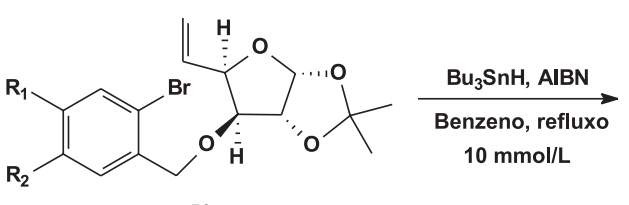

50

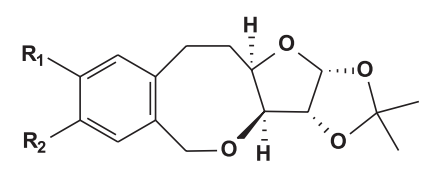

8-Endo (50-58\%)

a: R1=R2=H; b: R1=H, R2=OMe; $:$ R1=R2=OMe; d: R1+R2=OCH

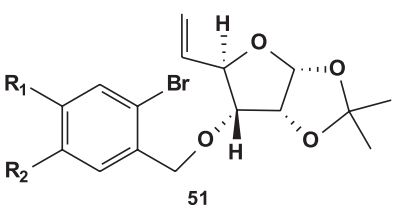

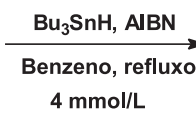

51

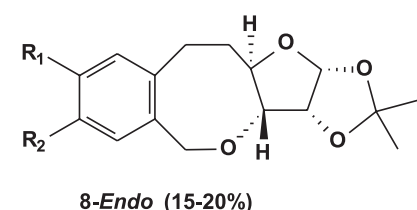

8-Endo $(15-20 \%)$

a: R1=R2=H; b: R1=H, R2=OMe; c: R1=R2=OMe; d: R1+R2=OCH

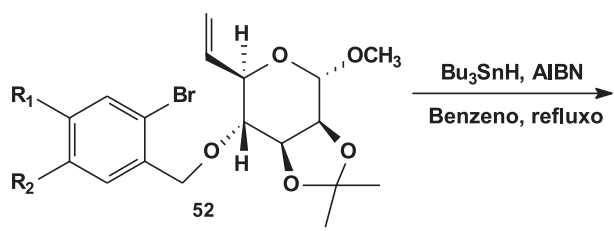<smiles>[R]c1cc2c(cc1[R2])C[OH+][C@H]1[C@@H](CC2)C[C@H](OC)[C@@H]2OC(C)(C)O[C@@H]21</smiles>

a: R1=R2=H; b: R1=H, R2=OMe; $:$ R1=R2=OMe; d: R1+R2=OCH

Esquema 31. Síntese de triciclos derivados de carboidratos

(Esquema 31), os rendimentos dos produtos de ciclização 8-endo variaram entre $60-75 \% .^{87}$

A síntese de peptídeos cíclicos de 14 e 17 membros, formados pelo modo endo, foi descrita por Balraju e colaboradores ${ }^{65}$ e envolveu reação de meta-bromobenzenos (53 e 54, Esquema 32) com $\mathrm{Bu}_{3} \mathrm{SnH}$ e AIBN em benzeno. Acredita-se que a existência de ligação de hidrogênio intramolecular no peptídeo acíclico entre o hidrogênio do N-H e o oxigênio da carbonila seja fundamental para a ciclização. Precursores do tipo 55, onde não há a possibilidade de formação deste tipo de ligação de hidrogênio intramolecular, mostraram-se incapazes de sofrer macrociclização nas mesmas condições de reação. ${ }^{65}$
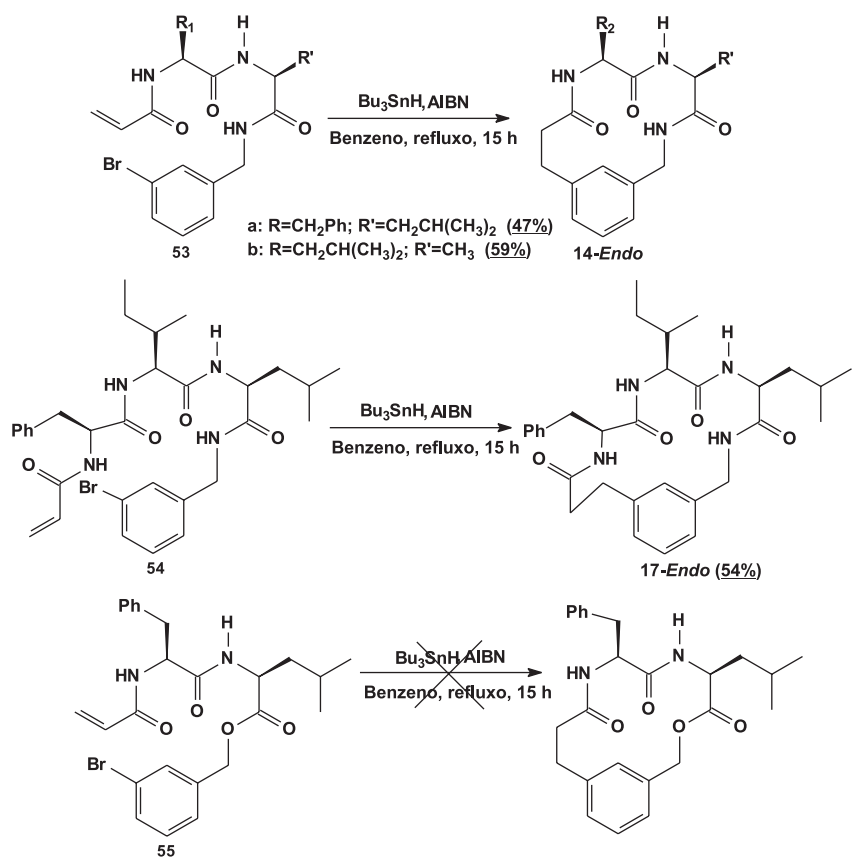

Esquema 32. Síntese de ciclopeptídeos por reação de carbociclização radicalar
No âmbito de um programa de pesquisa desenvolvido no Laboratório de Química Farmacêutica da Faculdade de Farmácia/UFMG, em parceria com o Departamento de Química do ICEx/UFMG (QF/DQ/UFMG), as sínteses de macrociclos, especialmente de benzomacrolactamas, foram planejadas com intuito de se verificar o papel de carboidratos e de sua estereoquímica em macrociclizações mediadas por $\mathrm{Bu}_{3} \mathrm{SnH}$, assim como a influência dos grupos envolvidos diretamente na reação de ciclização e de suas posições relativas na unidade sacarídica. O estímulo para o desenvolvimento deste trabalho é o desafio de sintetizar macrociclos, reconhecidamente um dos grandes problemas da síntese orgânica, ${ }^{1}$ aliado à possibilidade de obter substâncias com atividade biológica. Especialmente as benzomacrolactamas derivadas de carboidratos, maioria das substâncias sintetizadas no âmbito deste programa de pesquisa, são potenciais agentes biológicos, pois tratam-se de macrociclos, cujas atividades biológicas são em parte atribuídas à maior restrição conformacional em relação aos compostos de cadeia aberta, ${ }^{2,4}$ além de apresentarem quiralidade, anel aromático e grupo amida. Carey e colaboradores $^{5}$ relatam que, de 128 candidatos a fármacos que se encontram em desenvolvimento em três grandes indústrias farmacêuticas mais de $90 \%$ contém anel aromático, mais de $90 \%$ apresenta átomo de nitrogênio, 69 substâncias (54\%) são quirais e em 27 todos os centros estereogênicos são provenientes dos materiais de partida. Além disso, como dito anteriormente, $12 \%$ das reações realizadas para a síntese das 128 substâncias são de acilação, especialmente $N$-acilações. ${ }^{5}$

A primeira reação de carbociclização radicalar realizada no âmbito do programa de síntese de macrociclos pelo grupo de pesquisa QF/DQ/UFMG foi desenvolvida com a aliloxi-o-iodobenzamida de configuração glico 56 (Esquema 33), que conduziu à benzomacrolactama 11-endo 57 (40\%) e ao produto acíclico reduzido $58 .^{88} \mathrm{Em}$ seguida, a benzamida de configuração galacto 59 (Esquema 33), epímero em C-4 de 56, foi submetida à reação com $\mathrm{Bu}_{3} \mathrm{SnH}$ e conduziu à macrolactama 60, proveniente da ciclização 11-endo $(33 \%),{ }^{89}$ o que indicou que a configuração de C-4 do açúcar não interfere no modo pelo qual a macrociclização ocorre, nem significativamente no rendimento do produto de macrociclização. A formação dos macrociclos pelo modo endo já era prevista, uma vez que Porter e colaboradores estabeleceram o princípio de que "as macrociclizações endo são 

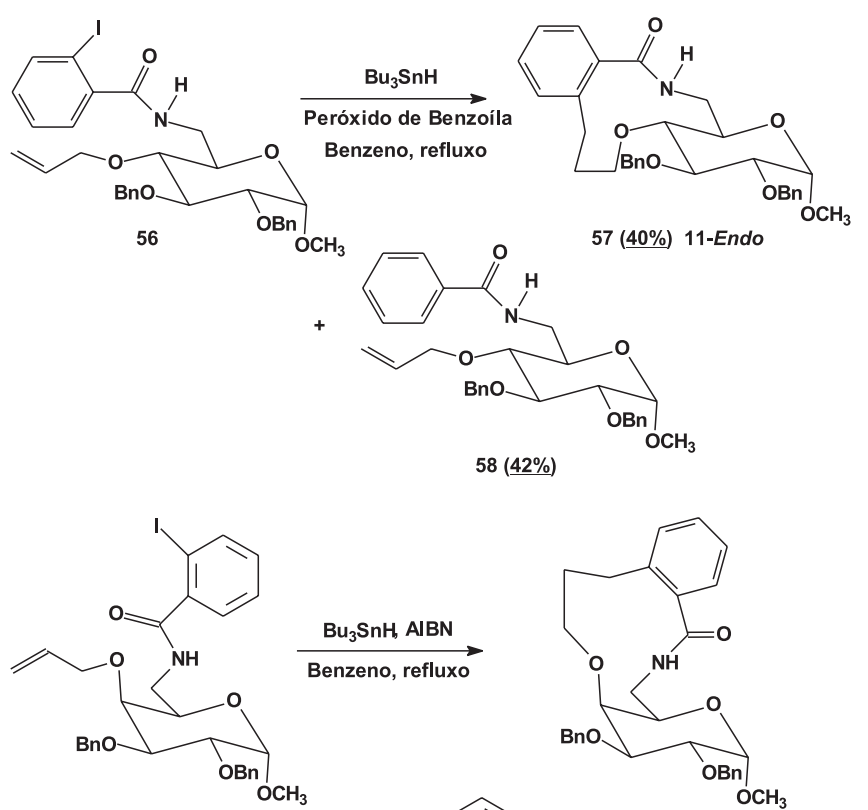

59

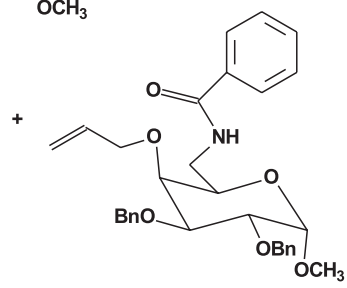

$61(\underline{22 \%})$

Esquema 33. Macrolactamas obtidas a partir de aliloxi-orto-iodobenzamidas

preferenciais em relação às exo", ${ }^{66-68}$ o que pode ser confirmado pelos trabalhos subseqüentes. ${ }^{69-87}$

Em outro estudo, ${ }^{88}$ a $o$-iodobenzamida 62 (Esquema 34), desprovida de unidade sacarídica, conduziu à macrolactama 63 (14\%), proveniente da ciclização 11-endo, mas com rendimento inferior aos das lactamas contendo unidade de açúcar. Este resultado demonstra a importância da restrição conformacional conferida pela presença do açúcar na macrociclização. ${ }^{88} \mathrm{As} o$-iodobenzamidas sem unidade sacarídica 65 e 67 (Esquema 34) foram submetidas à reação com $\mathrm{Bu}_{3} \mathrm{SnH} .{ }^{90}$ O substrato 67 levou à macrolactama de 12 membros 68 (19\%), mas da reação de $\mathbf{6 5}$, que poderia conduzir a um ciclo de 10 membros pelo modo endo, só foi obtido o produto de hidrogenólise $66 .{ }^{90}$ Considerando que as benzamidas 62 e 67, homólogas de $\mathbf{6 5}$, conduziram a produtos de ciclização endo, pode-se supor que as macrociclizações 11- e 12endo sejam mais favorecidas que a 10-endo. ${ }^{90}$

$\mathrm{Na}$ expectativa de que a presença de uma unidade de carboidrato pudesse conferir restrição conformacional ao substrato e favorecer a ciclização 10-endo, sintetizou-se a $o$-benzamida glico 70 (Esquema 35), que por reação com $\mathrm{Bu}_{3} \mathrm{SnH}$ conduziu à dilactama macrocíclia de 20 membros $\mathbf{7 1}$, formada por ataque intermolecular do radical arila ao carbono terminal do alqueno, seguido de macrociclização 20-endo. ${ }^{91}$ As aliloxi-o-iodobenzamidas 73 e 75, respectivamente, isômero de posição ( $O$-alil em C-2 e 2-iodobenzoilamino em C-3) e diastereoisômero (configuração altro) de $\mathbf{7 0}$ (Esquema 35), só conduziram aos respectivos produtos reduzidos não ciclizados 74 e 76. ${ }^{92}$ A princípio estes resultados confirmariam a dificuldade de ocorrência de ciclização 10-endo. Vale observar, no entanto, que os substituintes envolvidos na ciclização apresentam relação trans, o que pode ter contribuído para o insucesso das ciclizações. Embora haja relatos de ciclização em derivados de carboidratos com substituintes $\operatorname{trans},{ }^{87} \mathrm{em}$<smiles>C=CCOCCNC(=O)c1ccccc1I</smiles>

62

\section{$\mathrm{Bu}_{3} \mathrm{SnH}, \mathrm{AIBN}$}<smiles>C=CCOCCCNC(=O)c1ccccc1</smiles>

$64(\underline{85 \%})$
Benzeno, refluxo

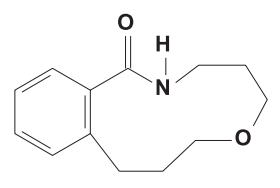

$63(\underline{14 \%}) 11-$ Endo<smiles>C=CCOCCNC(=O)c1cccc(OCC(C)C)c1</smiles><smiles>C[R16](C)(C)O</smiles><smiles>C=CCOCCCCNC(=O)c1ccccc1I</smiles>

$69(\underline{33 \%})$

Esquema 34. Orto-iodobenzamidas sem unidade sacarídica e produtos da reação com $\mathrm{Bu}_{3} \mathrm{SnH}$

outros substratos observou-se estereosseletividade nas ciclizações, que foram mais favorecidas nos substratos cis. $^{86}$

As benzamidas 77 e 80 (Esquema 36) conduziram aos macrociclos 11-endo $\mathbf{7 8}(17 \%)^{93}$ e $\mathbf{8 1}(8 \%),{ }^{94}$ respectivamente. Os rendimentos bem inferiores aos das macrolactamas formadas a partir de $\mathbf{5 6}(\mathbf{5 7}$ 40\%) e $\mathbf{5 9}(\mathbf{6 0} 33 \%)$ indicam a importância da posição dos grupos $O$-aliloxila (em C-4) e 2-iodobenzoilamino (em C-6) na porção sacarídica das iodobenzamidas de configuração glico $^{93}$ e galacto. ${ }^{94}$

Os aliloxi-o-iodobenzoatos 83 e $\mathbf{8 5}$ (Esquema 37) foram sintetizados e submetidos à reação de ciclização radicalar. ${ }^{95}$ Da reação do benzoato glico 83 só foi isolado o produto não ciclizado 84 . O substrato de configuração galacto $\mathbf{8 5}(500 \mathrm{mg})$ conduziu a $30 \mathrm{mg}$ de uma mistura contendo a macrolactama 11-endo 86 e ao produto de hidrogenólise 87 (Esquema 37). As diferenças observadas em relação às reações dos análogos nitrogenados 56 e 59 (Esquema 33) foram atribuídas à menor restrição conformacional em 83 e 85, tanto pelo fato de as rotações serem mais restritas nas amidas que nos ésteres, quanto pela possível formação em $\mathbf{5 6}$ e 59 de uma ligação de hidrogênio entre o hidrogênio amídico e o oxigênio do grupo aliloxila. ${ }^{95}$

Em outros trabalhos realizados ${ }^{96-100}$ foram sintetizados dois amidoésteres ( 88 e 91, Esquema 38) e quatro amidoéteres $(\mathbf{9 4 ,}, 97,99$ e 101, Esquema 38) contendo o grupo cinamila como alvo de ataque do radical arila, diferentemente dos trabalhos até então realizados, em que os substratos eram derivados alílicos. Esperava-se que quando submetidos à reação com $\mathrm{Bu}_{3} \mathrm{SnH}$ estes substratos levassem a macrociclos 11-endo (em geral mais favorecidos ${ }^{66-68}$ ) e/ou 10-exo. A ciclização 10-exo poderia ocorrer tanto pelo fato de os dois carbonos da ligação dupla serem substituídos, portanto do ponto de vista 

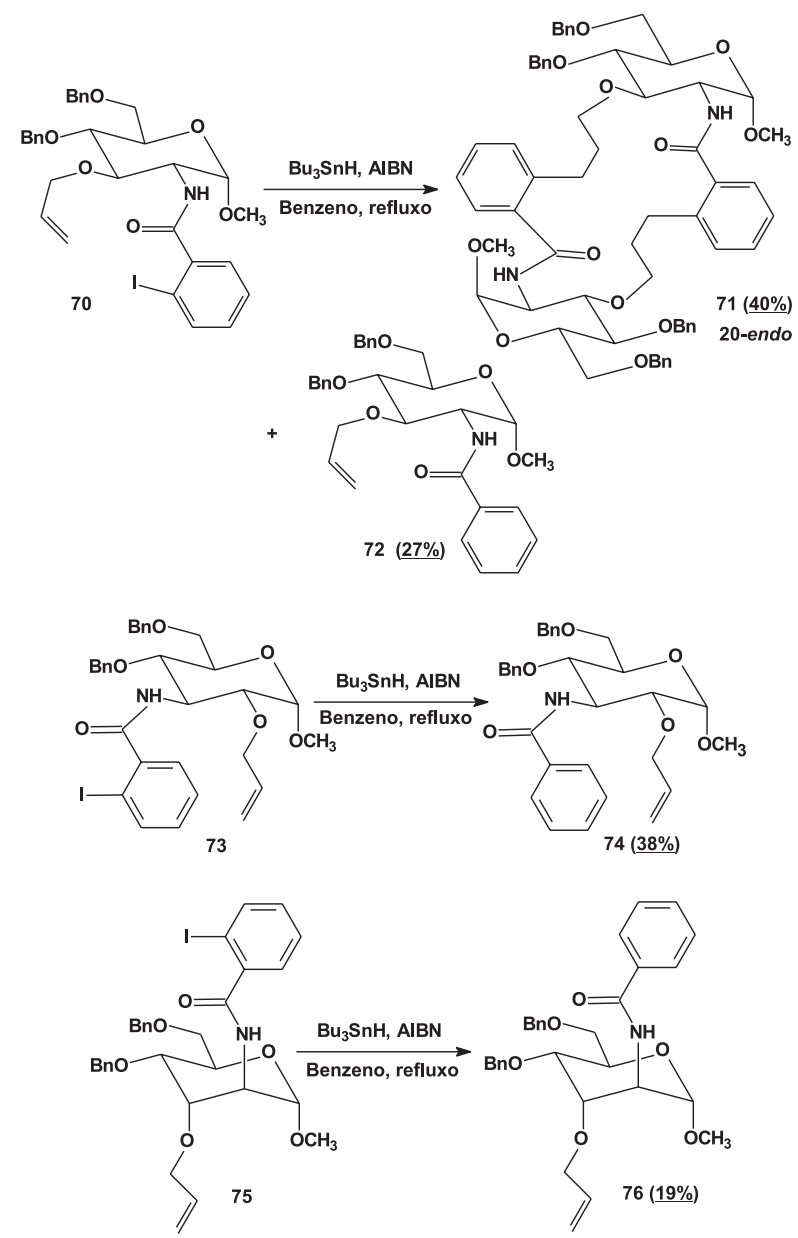

Esquema 35. Orto-iodobenzamidas glico e altro e produtos das reações com $\mathrm{Bu}_{3} \mathrm{SnH}$
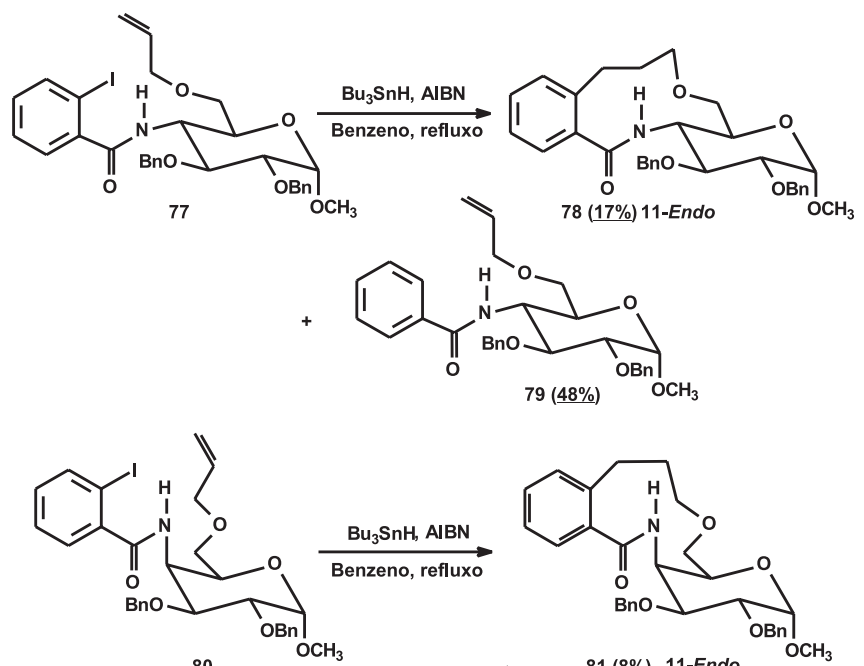

$81(8 \%)$ 11-Endo

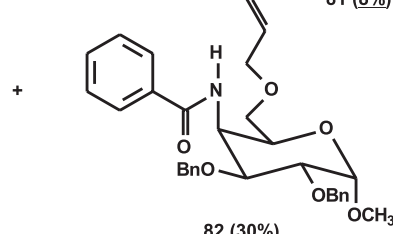

Esquema 36. Aliloxi-orto-iodobenzamidas glico e galacto e produtos das reações com $\mathrm{Bu}_{3} \mathrm{SnH}$
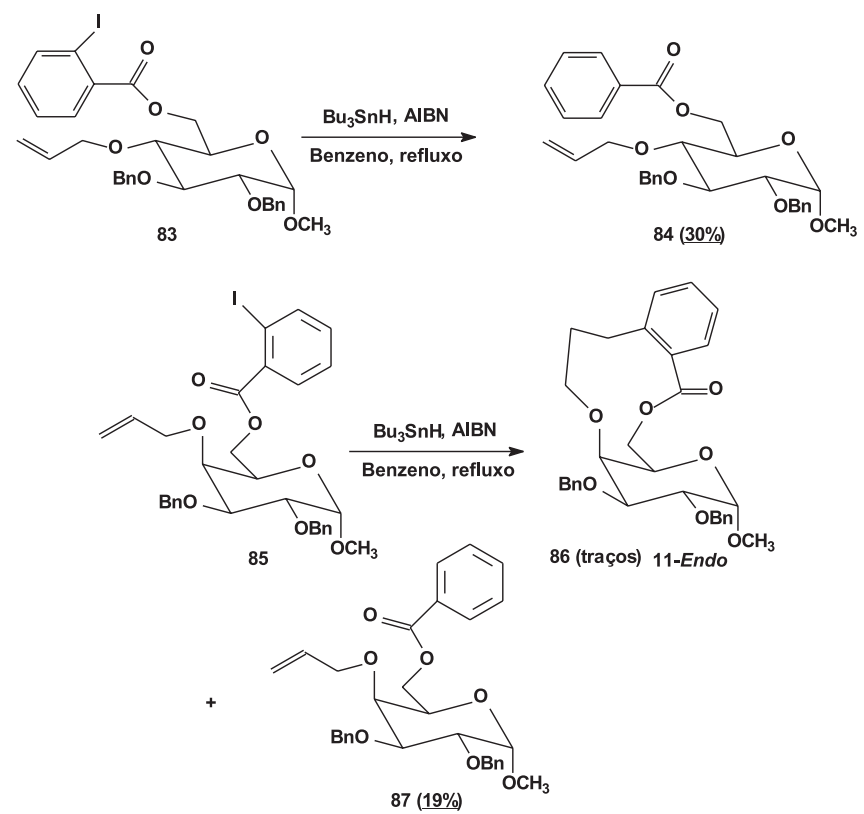

Esquema 37. Aliloxi-orto-iodobenzoatos e produtos das reações com $\mathrm{Bu}_{3} \mathrm{SnH}$

estérico serem aproximadamente equivalentes, quanto em função da estabilidade do radical benzílico que seria formado no caso do ataque 10-exo. Além disso, qualquer que fosse o modo de ciclização, 10-exo ou 11-endo, haveria formação de um novo centro estereogênico e seria possível verificar a estereosseletividade da ciclização. Das reações dos amidoésteres derivados do ácido cinâmico 88 e 91 com $\mathrm{Bu}_{3} \mathrm{SnH}$ foram isolados as imidas inesperadas $\mathbf{8 9}$ e $\mathbf{9 2}$, provenientes de ciclização 10-exo seguida de contração de anel, e os produtos de hidrogenólise 90 e 93, respectivamente. ${ }^{96}$ Nenhum dos amidoéteres cinamílicos (94, 97, 99 e 101) conduziu a produtos de ciclização quando submetidos à reação com $\mathrm{Bu}_{3} \mathrm{SnH}$. Foram isolados os respectivos produtos de hidrogenólise $(\mathbf{9 6}, \mathbf{9 8}, 100$ e 102) e da reação desenvolvida com 94 foi isolado também o derivado arilestanho $95 .{ }^{97-100} \mathrm{O}$ fato de não terem sido isolados produtos de macrociclização das reações desenvolvidas com os substratos amidoéteres cinamílicos $(\mathbf{9 4}, 97,99$ e 101, Esquema 38), ao contrário do que ocorreu com os respectivos amidoéteres $O$-alílicos (59, 56, 77 e 80, Esquemas 33 e 36), indica que o grupo fenila, possivelmente por impedimento estérico, dificulta o ataque do radical arila ao carbono mais externo da ligação dupla e não facilita o ataque 10-exo, reconhecidamente mais difícil. ${ }^{68}$

A amida 103 (Esquema 39), que apresenta o grupo 3-iodopropanoilamino em substituição ao 2-iodobenzoilamino em C-6 de 56, por reação com $\mathrm{Bu}_{3} \mathrm{SnH}$ conduziu majoritariamente ao produto de hidrogenólise 105 e à macrolactama $104(4 \%) .{ }^{101}$ Este resultado, comparado com o da reação radicalar da benzamida $\mathbf{5 6}$, demonstra que o ataque do radical arila é mais favorecido que o do radical alquila, possivelmente devido à maior liberdade conformacional do grupo alquila.

\section{NOVAS METODOLOGIAS PARA REAÇÃO DE CARBOCICLIZAÇÃO RADICALAR}

Uma metodologia que tem sido testada, mas que ainda está pouco desenvolvida, refere-se à condução de reações radicalares de moléculas pequenas em fase sólida, onde polímeros, como poliestireno, suportam precursores radicais. ${ }^{102-106}$

Em função de alguns inconvenientes que há na utilização do hidreto de tri- $n$-butilestanho, como toxicidade, dificuldade na separação 


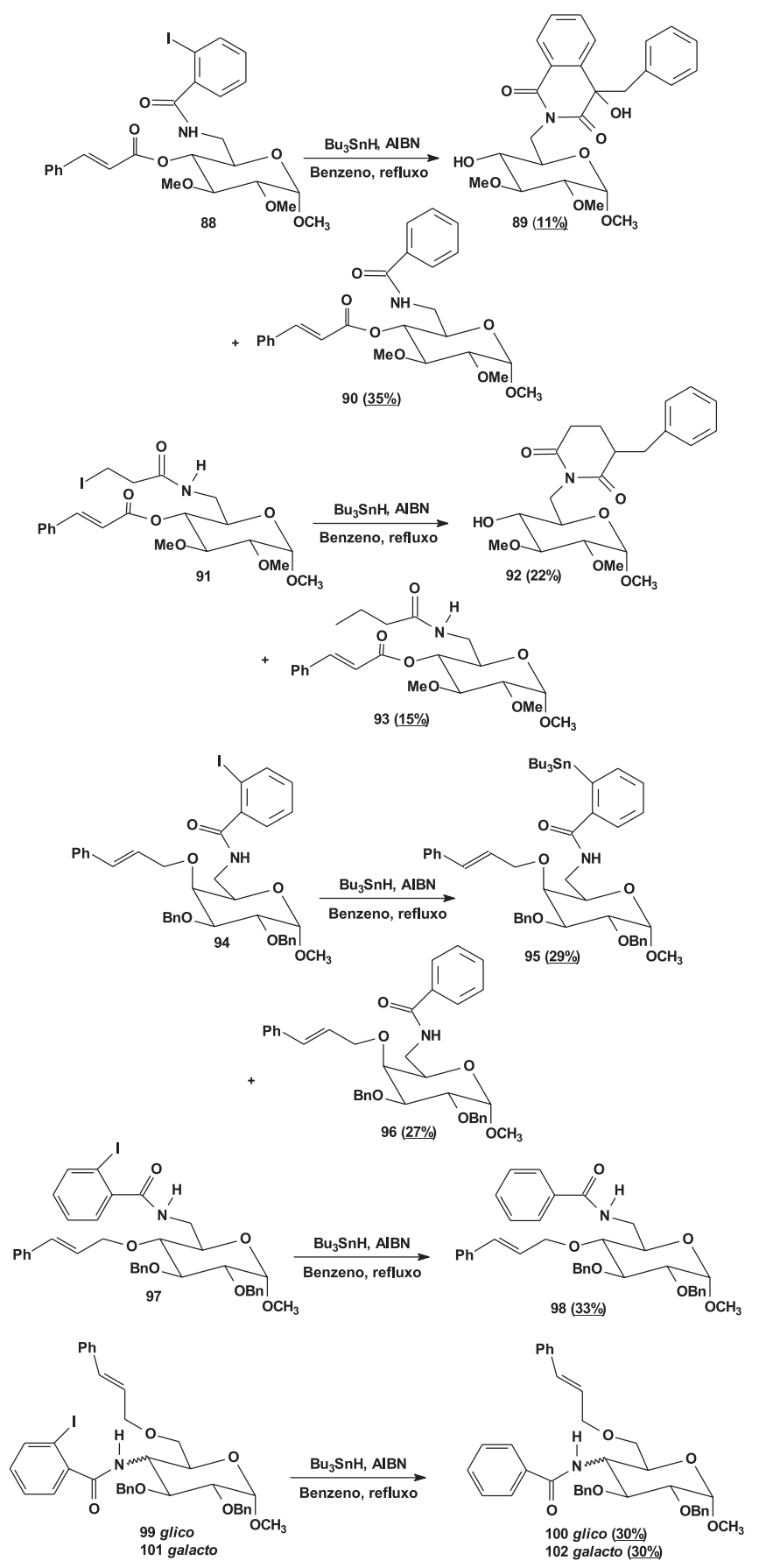

Esquema 38. Amidoésteres e amidoéteres cinamílicos e produtos das reações com $\mathrm{Bu}_{3} \mathrm{SnH}$

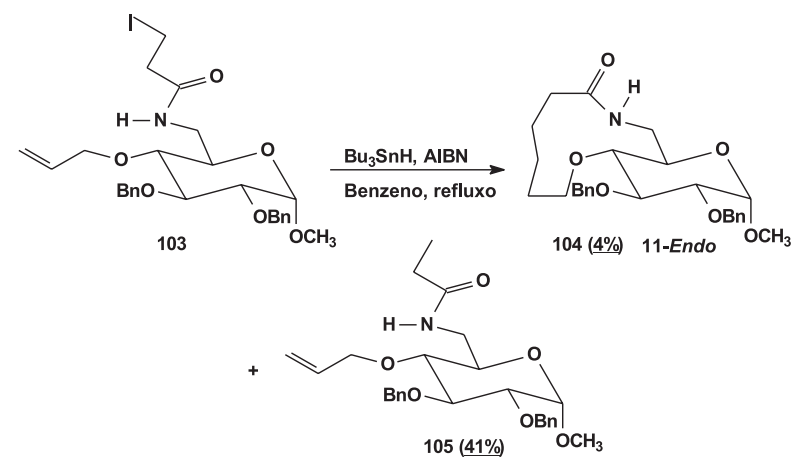

Esquema 39. Amida alquílica e produtos obtidos na reação com $\mathrm{B} u_{3} \mathrm{SnH}$ de resíduos de estanho do produto formado e redução do radical do substrato antes que ocorra a ciclização, ${ }^{56-58}$ alguns autores têm proposto utilização de reagentes alternativos para reações radicalares, como salofeno de sódio e cobalto (I), iodeto de samário (II) $\left(\mathrm{SmI}_{2}\right)$, tris(trimetilsilil)silano $\left(\left(\mathrm{Me}_{3} \mathrm{Si}\right)_{3} \mathrm{SiH}\right)$ e tris(trimetilsilil)germano $\left(\left(\mathrm{Me}_{3} \mathrm{Si}\right)_{3} \mathrm{GeH}\right) .{ }^{107,108}$

Outra alternativa que tem sido estudada mais recentemente, envolve formação de radicais induzidos por radiação ultravioleta (gerada, por exemplo, por lâmpada de mercúrio de alta pressão 150 W) para ciclização de radicais arila e piridila, conduzindo a anéis de 5 e 6 membros. ${ }^{109,110}$

Clyne e Aldabbagh ${ }^{58}$ realizaram um estudo mostrando rendimentos superiores na ciclização fotoquímica de radicais imidazólicos em relação à reação mediada por $\mathrm{Bu}_{3} \mathrm{SnH} / \mathrm{AIBN}$ para obtenção de ciclos de 6 membros. As principais vantagens da ciclização fotoquímica são: eliminação de resíduos tóxicos de estanho; eliminação do uso de iniciadores azo (AIBN), que são considerados perigosos; não há necessidade de adição lenta de $\mathrm{Bu}_{3} \mathrm{SnH} / \mathrm{AIBN}$, conseqüentemente o tempo total de reação é menor no caso da reação fotoquímica; o procedimento de elaboração e purificação é bem mais simples na reação fotoquímica, levando a bons rendimentos do produto ciclizado.

Ainda há poucos relatos de síntese de macrociclos utilizando reações fotoquímicas, ${ }^{111,112}$ sendo este um campo em aberto para pesquisas que podem ser promissoras e de grande utilidade para a química orgânica sintética.

\section{AGRADECIMENTOS}

Ao CNPq, pelas bolsas de mestrado, de produtividade em pesquisa e de iniciação científica.

\section{REFERÊNCIAS}

1. Blankenstein, J.; Zhu, J. P.; Eur. J. Org. Chem. 2005, 1949.

2. Ramaseshan, M.; Robitaille, M.; Ellingboe, J. W.; Dory, Y. L.; Deslongchamps, P.; Tetrahedron Lett. 2000, 41, 4737.

3. Wessjohann, L. A.; Ruijter, E.; Garcia-Rivera, D.; Brandt, W.; Mol. Diversity 2005, 9, 171.

4. Stachel, S. J.; Coburn, C. A.; Sankaranarayanan, S.; Price, E. A.; Pietrak, B. L.; Huang, Q.; Lineberger, J.; Espeseth, A. S.; Jin, L. X.; Ellis, J.; Holloway, M. K.; Munshi, S.; Allison, T.; Hazuda, D.; Simon, A. J.; Graham, S. L.; Vacca, J. P.; J. Med. Chem. 2006, 49, 6147.

5. Carey, J. S.; Laffan, D.; Thomson, C.; Williams, M. T.; Org. Biomol. Chem. 2006, 4, 2337.

6. Patel, V. F.; Andis, S. L.; Kennedy, J. H.; Ray, J. E.; Schultz, R. M.; J. Med. Chem. 1999, 42, 2588.

7. Wagner, B.; Gonzalez, G. I.; Dau, M. E. T. H.; Zhu, J.; Bioorg. Med. Chem. 1999, 7, 737.

8. Kohyama, N.; Yamamoto, Y.; Synlett 2001, 694.

9. Luesch, H.; Yoshida, W. Y.; Moore, R. E.; Paul, V. J.; Mooberry, S. L.; J. Nat. Prod. 2000, 63, 611.

10. Milligan, K. E.; Marquez, B. L.; Williamson, R. T.; Gerwick, W. H.; J. Nat. Prod. 2000, 63, 1440.

11. Nakagawa, Y.; Irie, K.; Nakamura, Y.; Ohigashi, H.; Bioorg. Med. Chem. Lett. 2001, 11, 723.

12. Shindo, K.; Kamishohara, M.; Odagawa, A.; Matsuoka, M.; Kawai, H.; J. Antibiot. 1993, 46, 1076.

13. Naruse, N.; Tenmyo, O.; Kawano, K.; Tomita, K.; Ohgusa, N.; Miyaki, T.; Konishi, M.; Oki, T.; J. Antibiot. 1991, 44, 733.

14. Martin, M.; Mas, G.; Urpi, F.; Vilarrasa, J.; Angew. Chem., Int. Ed. 1999, 38, 3086 .

15. Hegde, V. R.; Patel, M. G.; Gullo, V. P.; Ganguly, A. K.; Sarre, O.; Puar, M. S.; J. Am. Chem. Soc. 1990, 112, 6403. 
16. Hegde, V. R.; Patel, M. G.; Gullo, V. P.; Puar, M. S.; J. Chem.Soc., Chem. Commun. 1991, 810.

17. Hegde, V.; Patel, M.; Horan, A.; Gullo, V.; Marquez, J.; Gunnarsson, I.; Gentile, F.; Loebenberg, D.; King, A.; J. Antibiot. 1992, 45, 624.

18. Cooper, R.; Truumees, I.; Yarborough, R.; Loebenberg, D.; Marquez, J.; Horan, A.; Patel, M.; Gullo, V.; J. Antibiot. 1992, 45, 633.

19. Arai, T.; Suenaga, T.; Koyama, Y.; Honda, H.; J. Antibiot. 1962, 15, 231.

20. Bulusu, M. A. R. C.; Waldstatten, P.; Tricotet, T.; Rochais, C.; Steck, A.; Bacher M.; Tetrahedron Lett. 2004, 45, 5833.

21. Kawai, M.; Gunawardana, I. W. K.; Mollison, K. W.; Bioorg. Med. Chem. Lett. 1998, 8, 935.

22. Bornhovd, E.; Burgdorf, W. H. C.; Wollenberg, A.; J. Am. Acad. Dermatol. 2001, 45, 736 .

23. Dumont, F. J.; Curr. Med. Chem. 2000, 7, 731.

24. Wagner, R.; Mollison, K. W.; Liu, L. P.; Henry, C. L.; Rosenberg, T. A.; Bamaung, N.; Tu, N.; Wiedeman, P. E.; Or, Y.; Luly, J. R.; Lane, B. C.; Trevillyan, J.; Chen, Y. W.; Fey, T.; Hsieh, G.; Marsh, K.; Nuss, M.; Jacobson, P. B.; Wilcox, D.; Carlson, R. P.; Carter, G. W.; Djuric, S. W.; Bioorg. Med. Chem. Lett. 2005, 15, 5340.

25. Pattenden, G.; Thom, S. M.; Synlett 1993, 215.

26. Cheng, Y. Q.; Tang, G. L.; Shen, B.; J. Bacteriol. 2002, 184, 7013.

27. Szilagyi, A.; Fenyvesi, F.; Majercsik, O.; Pelyvas, I. F.; Bacskay, I.; Feher, P.; Varadi, J.; Vecsernyes, M.; Herczegh, P.; J. Med. Chem. 2006, 49, 5626.

28. Nishio, M.; Kohno, J.; Sakurai, M.; Suzuki, S.; Okada, N.; Kawano, K.; Komatsubara, S.; J. Antibiot. 2000, 53, 724.

29. Takatsu, T.; Ohtsuki, M.; Muramatsu, A.; J. Antibiot. 2000, 53, 1310.

30. Stead, P.; Latif, S.; Blackaby, A. P.; Sidebottom, P. J.; Deakin, A.; Taylor, N. L.; Life, P.; Spaull, J.; Burrell, F.; Jones, R.; Lewis, J.; Davidson, I.; Mander, T.; J. Antibiot. 2000, 53, 657.

31. Lemarchand, A.; Bach, T.; Tetrahedron 2004, 60, 9659.

32. Peng, W. M.; Blagg, B. S.; J. Org. Lett. 2006, 8975.

33. Bertasso, M.; Holzenkampfer, M.; Zeeck, A.; Stackebrandt, E.; Beil, W.; Fiedler, H. P.; J. Antibiot. 2003, 56, 364.

34. Kupchan, S. M.; Komoda, Y.; Thomas, G. J.; Smith, R. M.; Bryan, R. F.; Haltiwan, R. C.; Karim, A.; Court, W. A.; Gilmore, C. J.; J. Am. Chem. Soc. 1972, 94, 1354.

35. Yu, T.; Floss, H. G. Em Anticancer Agents from Natural Products; Cragg, G. M. L.; Kingston. D.; Newman, D. J., eds.; Taylor \& Francis: Boca Raton, 2005, cap. 17.

36. Widdison, W. C.; Wilhelm, S. D.; Cavanagh, E. E.; Whiteman, K. R.; Leece, B. A.; Kovtun, Y.; Goldmacher, V. S.; Xie, H. S.; Steeves, R. M.; Lutz, R. J.; Zhao, R.; Wang, L. T.; Blattler, W. A.; Chari, R. V. J.; J. Med. Chem. 2006, 49, 4392.

37. Trowitzsch, W.; Wray, V.; Gerth, K.; Hofle, G.; J. Chem. Soc., Chem. Commun. 1982, 1340.

38. Onishi, N.; Izaki, K.; Takahashi, H.; J. Antibiot. 1984, 37, 13.

39. Mack, M. M.; Molinski, T. F.; Buck, E. D.; Pessah, I. N.; J. Biol. Chem. 1994, 269, 23236.

40. Bailey, K. L.; Molinski, T. F; J. Org. Chem. 1999, 64, 2500.

41. Masuno, M. N.; Pessah, I. N.; Olmstead, M. M.; Molinski, T. F.; J. Med. Chem. 2006, 49, 4497.

42. Culvenor, C. C. J.; Beck, A. B.; Clarke, M.; Cockrum, P. A.; Edgar, J. A.; Frahn, J. L.; Jago, M. V.; Lanigan, G. W.; Payne, A. L.; Peterson, J. E.; Petterson, D. S.; Smith, L. W.; White, R. R.; Aust. J. Biol. Sci. 1977, 30, 269.

43. Koiso, Y.; Natori, M.; Iwasaki, S.; Sato, S.; Sonoda, R.; Fujita, Y.; Yaegashi, H.; Sato, Z.; Tetrahedron Lett. 1992, 33, 4157.

44. Li, P. X.; Evans, C. D.; Forbeck, E. M.; Park, H.; Bai, R. L.; Hamel, E.; Joullie, M. M.; Bioorg. Med. Chem. Lett. 2006, 16, 4804.
45. Gentile, G.; Fattori, D.; Botta, M.; Corelli, F.; Fusar-Bassini, D.; Lamba, D.; Can. J. Chem. 2000, 78, 925.

46. Ojima, I.; Geng, X. D.; Lin, S. N.; Pera, P.; Bernacki, R. J.; Bioorg. Med. Chem. Lett. 2002, 12, 349.

47. Jefferson, E. A.; Swayze, E. E.; Osgood, S. A.; Bioorg. Med. Chem. Lett. 2003, 13, 1635.

48. Frydman, B.; Bhattacharya, S.; Sarkar, A.; Drandarov, K.; Chesnov, S.; Guggisberg, A.; Popaj, K.; Sergeyev, S.; Yurdakul, A.; Hesse, M.; Basu, H. S.; Marton, L. J.; J. Med. Chem. 2004, 47, 1051.

49. Shan, D. D.; Chen, L.; Njardarson, J. T.; Gaul, C.; Ma, X. J.; Danishefsky, S. J.; Huang, X. Y.; Proc. Natl. Acad. Sci. U. S. A. 2005, 102,3772 .

50. Njardarson, J. T.; Gaul, C.; Shan, D.; Huang, X. Y.; Danishefsky, S. J.; J. Am. Chem. Soc. 2004, 126, 1038.

51. Gaul, C.; Njardarson, J. T.; Shan, D.; Dorn, D. C.; Wu, K. D.; Tong, W. P.; Huang, X. Y.; Moore, M. A.; Danishefsky, S. J.; J. Am. Chem. Soc. 2004, 126, 11326.

52. Handa, S.; Pattenden, G.; Contemp. Org. Synth. 1997, 4, 196.

53. Roxburgh, C. J.; Tetrahedron 1995, 51, 9767.

54. Marco-Contelles, J.; Alhambra, C.; Martínez-Grau, A.; Synlett 1998, 693.

55. Martinez-Grau, A.; Marco-Contelles, J.; Chem. Soc. Rev. 1998, 27, 155.

56. Berge, J. M.; Roberts, S. M.; Synthesis 1979, 471.

57. Salomon, C. J.; Danelon, G. O.; Mascaretti, O. A.; J. Org. Chem. 2000, 65, 9220.

58. Clyne, M.A.; Aldabbagh, F.; Org. Biomol. Chem. 2006, 4, 268.

59. Curran D. P.; Synthesis 1988, 417.

60. Walling, C.; Tetrahedron 1985, 41, 3887.

61. Beckwith, A. L. J.; Bowry, V. W.; Bowman, W. R.; Mann, E.; Parr, J.; Storey, J. M. D.; Angew. Chem., Int. Ed. 2004, 43, 95.

62. Snieckus, V.; Cuevas, J. C.; Sloan, C. P.; Liu, H.; Curran, D. P.; J. Am. Chem. Soc. 1990, 112, 896.

63. Giese, B.; Angew. Chem., Int. Ed. 1983, 22, 753.

64. Giese, B.; Radicals in Organic Synthesis: Formation of carboncarbon bonds, Pergamon Pres: Oxford, 1988.

65. Balraju V.; Reddy. D. S.; Periasamy, M.; Iqbal, J.; Tetrahedron Lett. 2005, 46, 5207.

66. Porter, N. A.; Magnin, D. R.; Wright, B. T.; J. Am. Chem. Soc. 1986, 108, 2787.

67. Porter, N. A.; Chang, V. H. T.; J. Am. Chem. Soc. 1987, 109, 4976.

68. Porter, N. A.; Chang, V. H. T.; Magnin, D. R.; Wright, B. T.; J. Am. Chem. Soc. 1988, 110, 3554.

69. Robertson, J.; Hatley, R. J. D.; Chem. Commun. 1999, 1455.

70. Hitchcock, S. A.; Pattenden, G.; J. Chem.Soc., Perkin Trans. 1 1992, 1323.

71. Cox, N. J. G.; Mills, S. D.; Pattenden, G.; J. Chem. Soc., Perkin Trans. 1 1992, 1313.

72. Robertson, J.; Burrows, J. N.; Stupple, P. A.; Tetrahedron 1997, $53,14807$.

73. Beckwith, A. L. J.; Drok, K.; Maillard, B.; Degueil-Castaing, M.; Philippon, A.; Chem. Commun. 1997, 499.

74. Begley, M. J.; Pattenden, G.; Smithies, A.; Tapolczay, D.; Walter, D. S.; J. Chem. Soc., Perkin Trans. 1 1996, 21.

75. Shea, K. J.; Odell, R.; Sasaki, D. Y.; Tetrahedron Lett. 1992, 33, 4699.

76. Baldwin, J. E.; Adlington, R. M.; Mitchell, M. B.; Robertson, J.; Tetrahedron 1991, 47, 5901.

77. Lamas, C.; Saa, C.; Castedo, L.; Dominguez, D.; Tetrahedron Lett. 1992, 33, 5653.

78. Jonas, D.; Ozlu, Y.; Parsons, P. J.; Synlett 1995, 255.

79. Houldsworth, S. J.; Pattenden, G.; Pryde, D. C.; J. Chem. Soc., Perkin Trans. 1 1997, 1091. 
80. Rodriguez, G.; Castedo, L.; Dominguez, D.; Saa, C.; Adam, W.; J. Org. Chem. 1999, 64, 4830.

81. Boger, D. L.; Mathvink, R. J.; J. Am. Chem. Soc. 1990, 112, 4008.

82. Astley, M. P.; Pattenden, G ; Synthesis 1992, 101.

83. Ishibashi, H.; Kawanami, H.; Nakagawa, H.; Ikeda, M.; J. Chem. Soc., Perkin Trans. 1 1997, 2291.

84. Ghosh, K.; Ghatak, U. R.; Tetrahedron Lett. 1995, 36, 4897.

85. Gibson, S. E.; Guillo, N.; Tozer, M. J.; Chem. Commun. 1997, 637.

86. Nandi, A.; Mukhopadhyay, R.; Chattopadhyay, P.; J. Chem. Soc., Perkin Trans. 1 2001, 3346.

87. Nandi, A.; Chattopadhyay, P.; Tetrahedron Lett. 2002, 43, 5977.

88. Prado, M. A. F.; Alves, R. J.; Souza, J. D.; Alves, R. B.; Pedrosa, M. T. C.; Prado, R. F.; Faraco, A. A. G.; J. Chem. Soc., Perkin Trans. 1 $2000,1853$.

89. Binatti, I.; Prado, M. A. F.; Alves, R. J.; Filho, J. D. S.; J. Braz. Chem. Soc. 2002, 13, 570.

90. Faraco, A. A. G.; Prado, M. A. F.; Alves, R. J.; Souza, J. D.; Alves, R. B.; Faraco, R. F. P.; Synth. Commun. 2003, 33, 463.

91. Faraco, A. A. G.; Prado, M. A. F.; Alves, R. B.; Faraco, R. F. P.; Alves, R. J.; Souza, J. D.; Meurer, E. C.; Eberlin, M. N.; Tetrahedron Lett. 2004, 45, 3317.

92. Binatti, I.; Tese de Doutorado, Universidade Federal de Minas Gerais, Brasil, 2005.

93. Dias, D. F.; Prado, M. A. F.; Pinto, G. D.; Alves, R. J.; Alves, R. B.; de Souza Filho, J. D.; Quim. Nova 2006, 29, 444.

94. Pires, M. C.; Prado, M. A. F.; Alves, R. J.; Resumos do XX Encontro Regional da Sociedade Brasileira de Química - MG, São João Del Rei, Brasil, 2006

95. Binatti, I.; Alves, R. B.; Prado, M. A. F.; Alves, R. J.; Souza-Filho, J. D.; Dias, D. F.; Quim. Nova 2005, 28, 1023.

96. De Oliveira, R. B.; De Souza, J. D.; Prado, M. A. F.; Eberlin, M. N.; Meurer, E. C.; Santos, L. S.; Alves, R. J.; Tetrahedron 2004, 60, 9901.
97. Oliveira, M. T.; Prado, M. A. F.; Alves, R.B.; Cesar, A.; Alves, R. J.; Queiroga, C. G.; Santos, L. S.; Eberlin, M. N.; J. Braz. Chem. Soc. 2007,18, 364 .

98. Rocha, A. P. C.; Prado, M. A. F.; Corrêa, C. E. M.; Resumos da XIV Semana de Iniciação Científica - Conhecimento e Cultura UFMG, Belo Horizonte, Brasil, 2005.

99. Pinto, G. D.; Prado, M. A. F.; Resumos da XIV Semana de Iniciação Científica - Conhecimento e Cultura - UFMG, Belo Horizonte, Brasil, 2005.

100. Queiroga, C. G.; Prado, M. A. F.; Resumos da XV Semana de Iniciação Científica - Conhecimento e Cultura - UFMG, Belo Horizonte, Brasil, 2006.

101. Rocha, A. P. C.; Prado, M. A. F.; Resumos do XX Encontro Regional da Sociedade Brasileira de Química - MG, São João Del Rei, Brasil, 2006.

102. Watanabe, Y.; Ishikawa, S.; Takao, G.; Toru, T.; Tetrahedron Lett. 1999, 40, 3411 .

103. Zhu, X. W.; Ganesan, A.; J. Comb. Chem. 1999, 1, 157.

104. Wendeborn, S.; De Mesmaeker, A.; Brill, W. K. D.; Acc. Chem. Res. 2000, 33, 215.

105. Caddick, S.; Hamza, D.; Wadman, S. N.; Wilden, J. D.; Org. Lett. 2002, 4, 1775.

106. Curran, D. P.; Yang, F. L.; Cheong, J. H.; J. Am. Chem. Soc. 2002, 124,14993

107. Harrowven D. C.; Sutton B. J.; Coulton S.; Org. Biomol. Chem. 2003, $1,4047$.

108. Bowman, W. R.; Fletcher, A. J.; Potts, G. B. S.; J. Chem. Soc., Perkin Trans. 1 2002, 2747.

109. Park, Y. T.; Jung, C. H.; Kim, M. S.; Song, N. W.; Kim, D.; J. Org. Chem. 2001, 66, 2197.

110. Park, Y. T.; Song, N. W.; Hwang, C. G.; Kim, K. W.; Kim, D.; J. Am. Chem. Soc. 1997, 119, 10677.

111. Griesbeck, A. G.; Oelgemoller, M.; Lex, J.; J. Org. Chem. 2000, $65,9028$.

112. Yoon, U. C.; Jin, Y. X.; Oh, S. W.; Cho, D. W.; Park, K. H.; Mariano, P. S.; J. Photochem. Photobiol., A 2002, 150, 77 